\title{
ESTRATÉGIA HÍBRIDA DE OTIMIZAÇÃO SEM DERIVADAS PARA A INVERSÃO COMPLETA DA FORMA DA ONDA
}

\author{
F. I. F. N. GOMES ${ }^{1,2^{*}}$, Y. S. F. BEZERRA ${ }^{1}$, M. V. C. HENRIQUES ${ }^{3}$, Á. BARROCA NETO ${ }^{1}$ E L. S. LUCENA ${ }^{1}$ \\ 'Universidade Federal do Rio Grande de Norte - UFRN \\ ${ }^{2}$ Instituto Federal de Educação, Ciência e Tecnologia do Rio Grande do Norte - IFRN \\ ${ }^{3}$ Universidade Federal Rural do Semi-Árido - UFERSA \\ iranildo.gomes@ifrn.edu.br*
}

Submetido 11/12/2016 - Aceito 10/05/2017

DOI: $10.15628 /$ holos.2017.5445

\section{RESUMO}

$\mathrm{Na}$ exploração sísmica, investiga-se as características de subsuperfície usando técnicas de inversão completa, da forma da onda (Full Waveform Inversion - FWI), a qual foi abordada como um problema de otimização não linear. A técnica FWI, tradicionalmente, usa métodos matemáticos baseados em derivadas e, portanto, falha quando a função objetiva é não diferenciável. Ademais, isso acarreta um alto custo computacional e uma precisão limitada a mínimos locais. Portanto, neste trabalho foi adotada uma metodologia sem derivadas, Derivative-Free Optimization (DFO), para encontrar o mínimo global. Neste tipo de abordagem, utiliza-se as técnicas Salto Aleatório (RJT), Busca Aleatória Controlada (CRS) e Simplex Adaptativo de Nelder-Mead (ANMS). Desenvolveu-se um algoritmo, FWI-DFO, que resolve numericamente a equação da onda acústica $2 \mathrm{D}$ pelo Método das Diferenças Finitas (FDM) e se utiliza de um método híbrido RJT-CRS-ANMS como técnica de otimização para a inversão sísmica. A estratégia é balancear automaticamente as buscas globais e locais iterativamente pelo CRS e ANMS, respectivamente. Aplicou-se a metodologia em cinco modelos reais de subsuperfície. Os resultados mostraram uma concordância significativa com os modelos reais. O tempo computacional apresentou valores razoáveis e a função objetivo mostrou ser bastante sensível a pequenas alterações nos parâmetros do modelo para os casos aqui analisados. Em síntese, a metodologia, FWIDFO, empregada mostrou-se bastante promissora na inversão sísmica.

\section{PALAVRAS-CHAVE: Inversão sísmica, Inversão completa da forma da onda, Otimização sem derivadas.}

\section{DERIVATIVE-FREE OPTIMIZATION HYBRID STRATEGY FOR FULL WAVEFORM INVERSION}

\section{ABSTRACT}

In the seismic exploration, the subsurface characteristics have been investigated using Full Waveform Inversion (FWI) techniques which was approached as a nonlinear optimization problem. The FWI technique traditionally uses mathematical methods based on derivatives and therefore fails when an objective function is non differentiable. In addition, this entails a high computational cost and a precision limited to local minimum. Therefore, in this work, a Derivative-Free Optimization (DFO) methodology was adopted to find the global minimum. In this type of approach, the Random Jump (RJT), Controlled Random Search (CRS) and Adaptive Nelder-Mead Simplex (ANMS) techniques were used. A FWI-DFO algorithm which numerically solves the $2 \mathrm{D}$ acoustic
\end{abstract}

wave equation by the Finite Differences Method (FDM) and uses a hybrid method RJT-CRS-ANMS as the optimization technique for the seismic inversion was developed. The strategy is to automatically balance global and local searches iteratively by CRS and ANMS, respectively. The methodology to five real subsurface models was applied. The results showed a significative agreement with the real models. The computational time presented reasonable values and the objective function showed to be very sensitive to small changes in the model parameters for the cases analyzed here. In summary, the FWI-DFO methodology proved to be very promising in the seismic inversion.

KEYWORDS: Seismic inversion, Full waveform inversion, Derivative-free optimization. 


\section{INTRODUÇÃO}

$\mathrm{Na}$ indústria do petróleo e gás, a exploração sísmica para caracterizar e identificar um reservatório (meio geológico) por meio de um modelo é um grande desafio para os pesquisadores dessa área de pesquisa. Isso se deve em grande parte à alta complexidade das estruturas geológicas do subsolo onde muitos reservatórios estão localizados. A ocorrência de heterogeneidades em todas as escalas e o reduzido conhecimento, antes de perfurações, das propriedades das diferentes partes do subsolo são algumas das causas das dificuldades encontradas no processo de exploração sísmica. Outra adversidade é devida à natureza dos dados obtidos nos levantamentos sísmicos e do resultado que se deseja para representar o meio geológico, denominado problema inverso sísmico (Martins, M. A., 2015 \& Rocha Jr. D. C., 2013).

$\mathrm{Na}$ busca por maior precisão das características da subsuperfície na exploração sísmica, novas técnicas de inversão foram desenvolvidas, como a Inversão Completa da Forma de Onda, Full Waveform Inversion (FWI). A modelagem FWI é uma técnica numérica para obtenção de parâmetros de modelos das propriedades da subsuperfície do meio; no caso das velocidades das rochas que compõem as camadas geológicas, a partir de um conjunto de dados sísmicos em que esses parâmetros são estimados por meio da resolução de um problema inverso. Geralmente, na resolução de problemas inversos sísmicos, o conjunto de dados advém de um levantamento sísmico em que, a partir de uma fonte sísmica, gera-se um campo de onda que se propaga por meio de um modelo de terra. Os efeitos de propagação, como reflexões, refrações, difrações, atenuações etc., são registrados por receptores posicionados na superfície da Terra. Esses registros (dados) são representados por sismogramas sintéticos ou reais. Isso objetiva utilizar todas as informações contidas nele, inclusive a fase e a forma da onda, tempo de trânsito que precisam ser processados e interpretados com o intuito de se identificar e caracterizar estruturas de subsuperfície das camadas do meio (Martins, 2015, Rocha Jr., 2013, Fichtner, A., 2011 \& Menke, W., 2012).

O processo de inversão está intrinsecamente relacionado ao problema direto. É por meio da solução do problema direto que se obtêm as respostas associadas ao modelo físico, uma vez conhecidos os parâmetros físicos que o definem, juntamente com a equação que descreve o fenômeno. Essas respostas (dados calculados) obtidas pelo problema direto são utilizadas no esquema de inversão que, por meio de um processo iterativo de otimização, buscam os parâmetros do modelo que, por sua vez, tentam replicar os dados registrados (dados observados) por algum aparato experimental (sismograma) (Martins, 2015).

O problema direto resolve o sistema de equações diferenciais parciais, que modela o fenômeno físico inúmeras vezes. Isso decorre do fato de se usar técnicas de otimização em que se define uma função custo (função objetivo) que quantifica o desajuste (misfit) entre os dados calculados e os observados a cada passo do algoritmo iterativo de otimização utilizado. Como consequência da minimização da função objetivo, os parâmetros do modelo tomados como verdadeiros são aqueles para os quais o desajuste entre os dados seja menor que um valor 
predeterminado (tolerância). Portanto, esses parâmetros caracterizam e identificam o modelo geológico pela inversão sísmica (Mrinal, K. S., 2006 \& Menke, W., 2012).

O FWI tradicionalmente usa métodos baseados em derivadas para otimizar a função custo. Isso apresenta um alto custo computacional e uma precisão limitada a mínimos locais. Portanto, para torná-lo mais robusto e competitivo, alguns autores utilizaram métodos de otimização livre de derivadas, Derivative-Free Optimization (DFO) (Bark, M. 2013, Conn, A. R., \& Vicente, L. N., 2009). Nessa perspectiva, o desenvolvimento e a aplicação de técnicas baseadas em DFO para otimizar o problema inverso sísmico são de grande interesse (Lima, M.V.A.G., 2006, DinizEhrhardt, M. A., 2010).

Dentre as várias técnicas DFO, existem aquelas que se enquadram na classe de métodos de busca direta. São métodos iterativos em que um conjunto de pontos ou modelos é testado a cada iteração, adotando estratégias que usam somente avaliações da função objetivo para definir a aproximação seguinte. Os métodos baseados em DFO geralmente são bem-sucedidos, robustos, fáceis de implementar e, hoje, representam uma alternativa em ocasiões nas quais outros métodos podem falhar. Isto pode ocorrer pela impossibilidade, proibição ou dificuldade de se calcular as derivadas necessárias ao problema (Lima, 2006, Diniz-Ehrhardt, M.A., Martinez, J. M. \& Pedroso, L.G., 2011, Gao, F. \& Han, L., 2012). Portanto, neste trabalho, foi adotada uma metodologia híbrida de otimização com base em DFO.

O objetivo específico desta pesquisa é a elaboração de um algoritmo FWI-DFO para resolver o problema inverso sísmico aplicado a cinco modelos geológicos. A modelagem direta resolve numericamente a equação da onda acústica sísmica que simula a propagação em duas dimensões (2D) no domínio do tempo usando o Método das Diferenças Finitas, Finite Difference Method (FDM). A técnica DFO, que usa uma estratégia híbrida com base nos métodos Técnica do Salto Aleatório, Random Jump Technique (RJT), Busca Aleatória Controlada, Controlled Random Search (CRS), o método de busca Simplex Adaptativo de Nelder-Mead, Adaptive Nelder-Mead Simplex (ANMS), foi utilizada no processo de otimização que resolve o problema inverso sísmico. Isto caracteriza uma modelagem $\mathrm{FWI}$ com estratégia híbrida de otimização com base em DFO= RJT-CRS-ANMS.

\section{FORMULAÇÃO MATEMÁTICA DO FWI-DFO}

Nesta seção, serão revistos, brevemente, alguns aspectos da modelagem FWI aplicada ao Problema Inverso Sísmico (PIS). Basicamente, a técnica FWI constitui-se nos passos descritos a seguir. Com base nos parâmetros de modelos estimados pela inversão, podem-se predizer os dados (dados calculados) resolvendo o Problema Direto Sísmico (PDS). O PDS que descreve o fenômeno de propagação da onda acústica em um meio contínuo provocado por uma fonte sísmica foi resolvido numericamente pelo Método das Diferenças Finitas (FDM). Dessa forma, podem-se comparar os dados calculados com os dados observados do experimento sísmico, 
registrados em sismogramas (reais ou sintéticos), utilizando a norma $l_{2}$ como medida para quantificar a distância entre eles. Essa medida constitui a função custo ou função objetivo do PIS. Nesta pesquisa, o PIS utiliza-se de um método de otimização livre de derivada (DFO) para minimizar essa função objetivo e encontrar o melhor conjunto de parâmetros de modelo que se ajuste aos dados reais, caracterizando e identificando, assim, o meio geológico.

\subsection{O problema inverso sísmico (PIS)}

Pode-se definir o PIS ou inversão como um conjunto de técnicas matemáticas para extrair de dados observados (registro de efeitos) os parâmetros físicos correspondentes ao sistema, considerando um modelo físico-matemático que explique a relação entre ambos (Almeida, $V . X$. D., 2013). Abordando-se matematicamente a teoria da inversão, define-se: d como o vetor de dados; $\mathbf{m}$ como o vetor de parâmetros do modelo (ou apenas modelo), e $G$ é um operador matemático (equações governantes) que relaciona os parâmetros do modelo $\mathbf{m}$ aos dados $\mathbf{d}$ (i.e. a física governante). Estas grandezas estão relacionadas pela equação:

$$
\mathbf{d}=G(\mathbf{m})
$$

Logo, o PIS consiste em: tendo-se d e $G$, obtém-se m. O PIS constitui um processo iterativo de ajuste de dados que se propõe a fornecer informações quantitativas, do campo de velocidades da subsuperfície. Esse processo é baseado na modelagem do campo de ondas completo, e tem como objetivo minimizar a diferença entre os dados observados (registrados em sismogramas pela exploração sísmica) e os dados calculados em um modelo inicial de parâmetros, denominado desajuste (misfit). (Rocha Jr., D. C., 2013 \& Tarantola, A., 2006). O desajuste é quantificado pelo quadrado da norma do vetor desajuste.

Neste trabalho, a função objetivo $\phi$, desajuste, utilizada no procedimento de inversão, quantifica a diferença entre as amplitudes calculadas e observadas da onda compressional refletida (onda acústica, onda $\mathrm{P}$ ). Portanto, no contexto da técnica de mínimos quadrados, $\mathrm{O}$ melhor ajuste dos dados calculados aos observados está associado ao valor mínimo de $\phi$, dado pela Equação (2) (Lima, 2006):

$$
\phi=\sum_{j} \sum_{k}\left(A_{j, k}^{o b s}-A_{j, k}^{c a l}\right)^{2}
$$

Dado este em que o índice $j$ refere-se ao número do traço registrado; o índice $k$ referese ao número da amostra dentro da janela de tempo; $\mathbf{A}^{\text {obs }}$ são as amplitudes observadas no sismograma registrado; $\mathbf{A}^{\text {calc }}$ são as amplitudes do sismograma sintético calculado em função dos parâmetros do modelo $\left(V_{i}, h_{i}\right)$ que correspondem à velocidade da onda na i-ésima camada e à profundidade do i-ésimo refletor, respectivamente. A Figura 1 (b) mostra o esquema do PIS.

\subsection{0 problema direto sísmico (PDS)}


O PDS consiste na determinação do campo de propagação da onda a partir de um conjunto de parâmetros (parâmetros de modelo) que caracterizam o meio geológico, por meio da equação matemática (equação da onda) que relaciona os parâmetros do modelo aos dados (dados calculados). Com isso, é possível prever se os dados observados, registrados em um sismograma, correspondem a certo modelo que represente o meio geológico. Essas respostas (dados calculados) serão utilizadas em um esquema de inversão que busca identificar os parâmetros do modelo que descrevem com a melhor aproximação possível os dados observados. Portanto, a formulação do problema direto e o processo de obtenção de suas soluções consistem nas primeiras tarefas a serem realizadas em qualquer abordagem de problemas inversos (Martins, 2015 \& Lima, 2006). Logo, o PDS consiste em: tendo-se $\mathbf{m}$ e $G$, obtém-se d pela Equação (1). A Figura 1 (a) mostra o esquema do PDS.

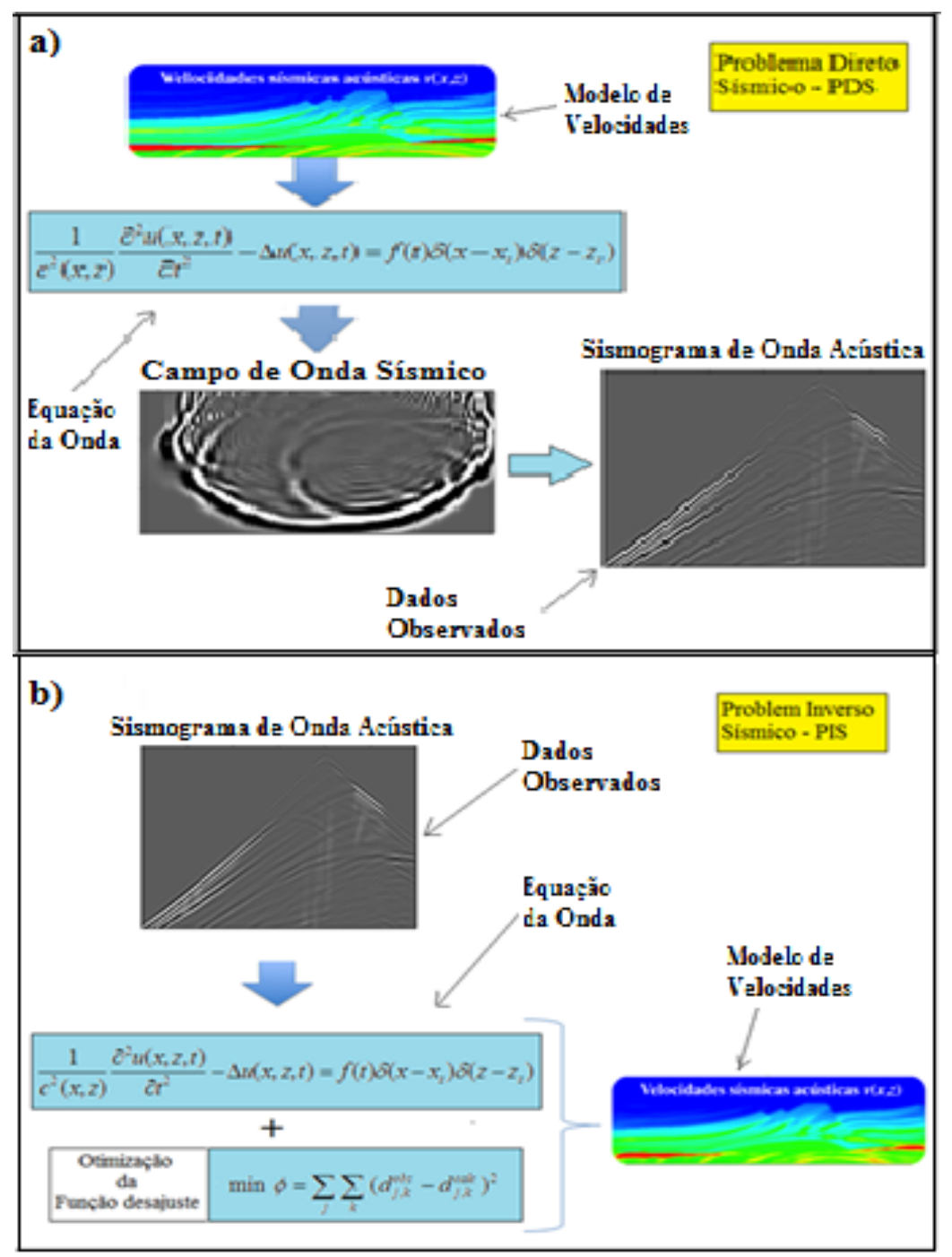

Figura 1: (a) Esquema do Problema Direto Sísmico (PDS); (b) Problema Inverso Sísmico (PIS).

Fonte: Adaptado de Martins, M.A. (2015). Estratégias para inversão do campo de ondas completo associado a fontes sísmicas reais e virtuais. (Tese de doutorado - Universidade Federal do Rio de Janeiro). 
A técnica FWI é vista como um problema de otimização não linear, mais precisamente um problema de minimização de um funcional (o quadrado da norma $l_{2}$ do resíduo). Tradicionalmente, o FWI constitui-se como um processo de otimização de busca local. Para resolver esse tipo de problema, usam-se métodos iterativos que usam derivadas e um único ponto de partida - ver Figura 2 (a). Entretanto, existem métodos iterativos de otimização livre de derivadas (Derivative-Free Optimization - DFO), que não exigem a especificação de um único ponto de partida e que podem escapar de mínimos locais. Para melhorar o processo de busca pelo ótimo global - ver Figura 2 (b) -, a proposta desta pesquisa é substituir o processo de otimização tradicional do FWI por uma metodologia híbrida baseada em DFO.
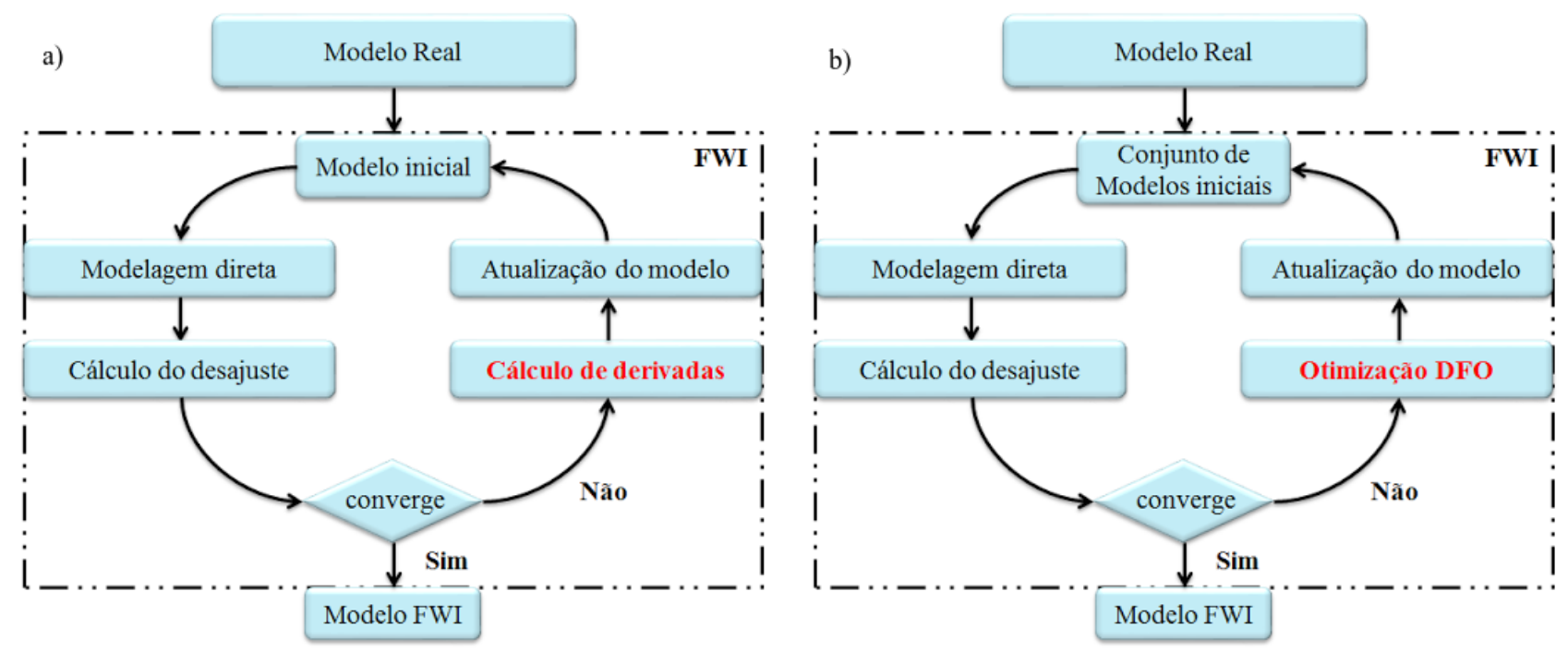

Figura 2: Fluxograma ilustrativo da metodologia (a) FWI tradicional; (b) FWI - DFO.

A metodologia FWI tradicional pode ser sintetizada na forma do fluxograma da Figura 2 (a), enquanto que a modelagem FWI-DFO é sintetizada na Figura 2 (b).

\subsection{Modelagem 2D}

\subsubsection{A equação de onda acústica}

O problema direto corresponde ao modelo matemático da propagação da onda acústica, equação da onda (EO), em duas dimensões (2D), que, juntamente com suas condições iniciais (Cl) e condições de contorno (CC), é dado pelo operador matemático $\Im(m)=u$ representado pelo conjunto de Equações (3A, 3B, 3C e 3D) como segue: 


$$
\Im(m)=u\left\{\begin{array}{l}
\text { (EO) } \frac{1}{c^{2}(x, z)} \frac{\partial^{2} u(x, z, t)}{\partial t^{2}}-\Delta u(x, z, t)=f(t) \delta\left(x-x_{s}\right) \delta\left(z-z_{s}\right) \\
\text { (CI) } u(x, z, t)=0, \quad \text { para } t \leq 0
\end{array}\right\}\left\{\begin{array}{l}
\frac{1}{c\left(x_{0}, z\right)} \frac{\partial u\left(x_{0}, z, t\right)}{\partial t}-\frac{\partial u\left(x_{0}, z, t\right)}{\partial x}=0 \Rightarrow L F S \\
\frac{1}{c\left(x_{n x}, z\right)} \frac{\partial u\left(x_{n x}, z, t\right)}{\partial t}+\frac{\partial u\left(x_{n x}, z, t\right)}{\partial x}=0 \Rightarrow R H S \\
\frac{1}{c\left(x, z_{0}\right)} \frac{\partial u\left(x, z_{0}, t\right)}{\partial t}-\frac{\partial u\left(x, z_{0}, t\right)}{\partial z}=0 \Rightarrow \text { TOP } \\
\frac{1}{c\left(x, z_{n z}\right)} \frac{\partial u\left(x, z_{n z}, t\right)}{\partial t}+\frac{\partial u\left(x, z_{n z}, t\right)}{\partial z}=0 \Rightarrow B O T
\end{array}\right\} \text { Direção } x \text { (3C) }
$$

sendo $c$ a velocidade de propagação da onda, $u$ o campo de amplitudes, ou pressão. $\delta\left(x-x_{s}\right) \mathrm{e}$ $\delta\left(z-z_{s}\right)$ são distribuições conhecidas por função delta de Dirac. Elas têm a finalidade de localizar espacialmente a função fonte sísmica $f(t)$ na posição $\left(x_{s}, z_{s}\right)$. Este é um pulso que é usualmente aproximado pela derivada da gaussiana, conhecida como wavelet de Ricker (Ricker, N. H., 1940). É da forma

$$
f(t)=\left(1-2 \pi^{2} v_{0}^{2}\left(t-t_{0}\right)^{2}\right) e^{-\pi^{2} v_{0}^{2}\left(t-t_{0}\right)^{2}}
$$

sendo

$$
t_{0}=\frac{6}{\pi v_{0} \sqrt{2}}
$$

com $t$ a janela do tempo $(s)$ utilizada na amostragem sísmica, $v_{0}(\mathrm{~Hz})$ é a frequência de corte ou de pico e $\left(t-t_{0}\right)$ é o tempo defasado. $\mathrm{E} \Delta$, o operador Laplaciano, dado pela Equação (5):

$$
\Delta u(x, z, t)=\frac{\partial^{2} u(x, z, t)}{\partial x^{2}}+\frac{\partial^{2} u(x, z, t)}{\partial z^{2}}
$$

Para as CC, utiliza-se a Condição de Bordas Absorventes, Absorbing Boundary Conditions $(A B C)$. Isso aumenta a eficiência computacional, permitindo, assim, modelar um meio infinito além 
das bordas do modelo geológico de interesse (Fichtner, A., 2011). A equação correspondente advém da hipótese feita por d'Alembert para a solução analítica da equação da onda 1D. Em 2D, utiliza-se a mesma solução referente ao caso de uma dimensão 1D, para a direção $x$ (esquerda LFT e direita - RHS), e $z$ (topo - TOP e fundo - BOT), respectivamente, para o caso 2D (Reynolds, A. C., 1978).

\subsubsection{Discretização pelo método das diferenças finitas (FDM)}

O problema direto é descrito por sistemas de Equações Diferenciais Parciais (EDP) equação da onda - (EO). Geralmente, não existem soluções analíticas para ela. Então, lançam-se mãos de técnicas numéricas para resolvê-la aproximadamente. Existem vários métodos que podem ser usados para este fim: o Método dos Elementos Finitos, Finite Elements Method (FEM), o Método das Diferenças Finitas (FDM), entre outros. Neste trabalho, utilizam-se o Método das Diferenças Finitas, Finite Difference Method (FDM). A essência desse método numérico consiste em um esquema de discretização, que transforma o domínio contínuo $u^{t}(x, z)$ em um domínio discreto $U_{i j}^{t}$, sendo $u^{t}\left(U^{t}\right)$ a solução da EO em cada ponto do domínio considerado no nível de tempo $t$. Este procedimento leva a construção de uma malha de pontos nodais $U_{i j}^{t}$, geralmente, regularmente espaçados de $\Delta x=\Delta z=h$ em cada direção coordenada (graus de liberdade do sistema). O critério de estabilidade deve ser satisfeito, critério de Courant-Friedrichs-Lewy ou condição CFL, para que o sistema possa convergir para a solução desejada. Ver Equação (6) abaixo:

$$
\Delta t \leq \alpha \frac{h}{C_{\max }} \quad \text { com } \quad \alpha=1 / 6 \quad(\text { Critério }-C F L)
$$

Equação esta em que $\Delta t$ é o passo do tempo, $h=\Delta x=\Delta z$ o espaçamento da malha regular e uniforme, e $C_{\max }$ é a velocidade máxima de propagação da onda no modelo de velocidades.

As derivadas de segunda ordem, espaciais e temporais na EO, foram substituídas por fórmulas centradas de segunda ordem. Ou seja, trata-se de um esquema padrão de cinco pontos em 2D com precisão de segunda ordem (Cunha, M. C. C., 2000, Alford, R., Kelly, K. \& Boore, D., 1974), sendo um Stencil de cinco pontos (Dourado, W. B., Imada, R. N. \& Pagamisse, A., 2013). Utiliza-se uma malha regular e uniforme, cuja grade cartesiana é de tamanho $n_{x} \times n_{z}$, enquanto as derivadas de primeira ordem foram substituídas por diferenças finitas avançadas no tempo, de primeira ordem, e avançadas e atrasadas no espaço de segunda ordem nas bordas esquerda e direita (topo e fundo), respectivamente (Cunha, 2000).

Então, discretizando as EO, Equações (3A e 3B), e as bordas CC-ABS, Equações (3C e 3D), Isolando a variável de interesse, $U_{i j}^{t+1}$, nestas equações, têm-se um esquema explícito para o FDM, que fornece os valores de $U_{i j}$ no tempo futuro $t+1$ em função de $U_{i j}$, no tempo presente, $t$ e no tempo passado $t-1$, para cada passo de tempo $\Delta t$, ou seja: 


$$
\operatorname{Bordas}(x)\left\{\begin{array}{l}
U_{1 j}^{t+1}=\left(1-3 / 2 \lambda_{1 j}\right) U_{1 j}^{t}+2 \lambda_{1 j} U_{2 j}^{t}-1 / 2 \lambda_{1 j} U_{3 j}^{t}, \quad j=1, \ldots, n z \\
U_{n x j}^{t+1}=\left(-1 / 2 \lambda_{n x j}\right) U_{n x-2 j}^{t}+2 \lambda_{n x j} U_{n x-1 j}^{t}\left(1-3 / 2 \lambda_{n x j}\right) U_{n x j}^{t}, \quad j=1, \ldots, n z
\end{array}\right.
$$

Pontos internos $\left\{U_{i j}^{t+1}=2 U_{i j}^{t}+\lambda_{i j}^{2} \Delta^{*} U_{i j}^{t}-U_{i j}^{t-1}+\sigma_{i j}^{2} f_{i j}^{t}, \quad i=2, \ldots, n x-1\right.$ e $\quad j=2, \ldots, n z-1$ (7C)

$$
\operatorname{Bordas}(z)\left\{\begin{array}{l}
U_{i 1}^{t+1}=\left(1-3 / 2 \lambda_{i 1}\right) U_{i 1}^{t}+2 \lambda_{i 1} U_{i 2}^{t}-1 / 2 \lambda_{i 1} U_{i 3}^{t}, \quad i=1, \ldots, n x \\
U_{i n z}^{t+1}=\left(-1 / 2 \lambda_{i n z}\right) U_{i n z-2}^{t}+2 \lambda_{i n z} U_{i n z-1}^{t}\left(1-3 / 2 \lambda_{i n z}\right) U_{i n z}^{t}, \quad i=1, \ldots, n x
\end{array}\right.
$$

Este sistema de equações lineares representa um esquema de evolução temporal da variável $U$ a cada nível de tempo $t+1$, de modo explícito.

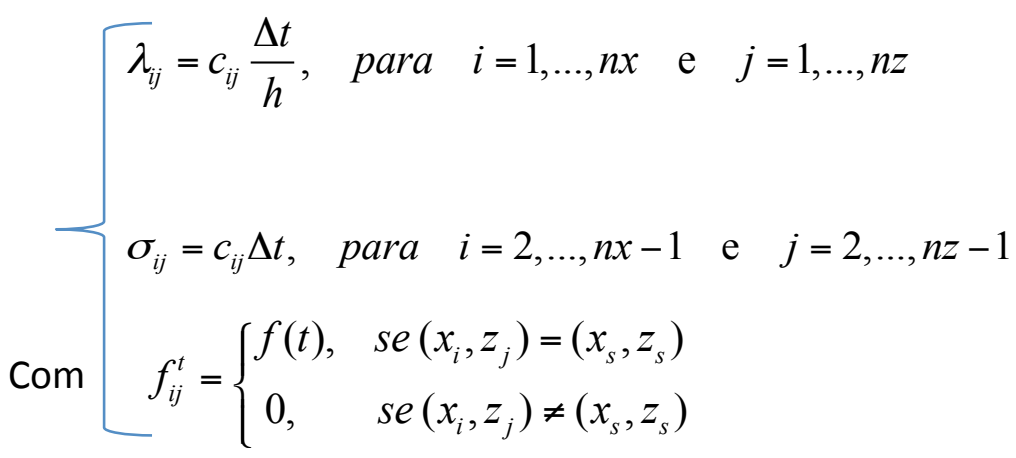

\subsection{Problema de otimização}

Nos problemas de reflexão sísmica, a determinação de parâmetros de subsuperfície é feita por meio da inversão dos coeficientes de reflexão da onda $P$ refletida. Sendo assim, um problema de otimização não linear consiste em encontrar o vetor de parâmetros $m=\left[m_{1}, \ldots, m_{M}\right]$ de modo que a função objetivo $\phi(\mathbf{m})$ seja minimizada (Lima, 2006). Neste contexto, o problema da inversão é naturalmente formulado como um problema de otimização. Este problema constitui um processo iterativo de ajuste de dados, em que, a cada etapa, atualiza-se o conjunto de parâmetros do modelo. Existem duas abordagens para resolver este problema, uma delas baseada no cálculo das derivadas da função. Esses métodos tendem a convergir para um ótimo local (mínimo local), e sua eficácia depende de quão próximo o ponto inicial esteja desse ótimo. A outra abordagem usa métodos de otimização livre de derivadas (DFO) e não exige a especificação de um único modelo inicial, que possa escapar de mínimos locais tais como: a Técnica do Salto Aleatório (RJT), a Busca Aleatória Controlada (CRS), o método de busca Adaptativa de Nelder-Mead (ANMS), o método de Monte Carlo (MC) etc. (Bakr, M., 2013, Gao \& Han, 2012). 
Existem várias técnicas de otimização com base em DFO. Algumas delas são puramente estocásticas (RJT, MC), outras baseiam-se no processo denominado de busca direta (CRS, ANMS) com características estocásticas e evolucionárias. Estes são métodos populacionais em que uma população inicial de pontos é melhorada de uma maneira iterativa. O processo concentra-se na exploração direta do espaço de busca, em que se procura a direção de melhor resultado para minimizar a função custo do problema (Tran, K. T. \& Hiltunen, D, R., 2012). As técnicas DFO podem classificar-se como métodos de mínimo local, ou de mínimo global, dependendo da estratégia empregada para se encontrar o ponto ótimo.

Mais precisamente, a etapa da otimização (minimização) do Problema da Inversão Sísmica (PIS) pode ser tratada como um Problema de Programação Não Linear (PPNL). Na forma canônica um PPNL se escreve como:

$$
\begin{aligned}
& \min \quad \phi=\sum_{j} \sum_{k}\left(A_{j, k}^{\text {obs }}-A_{j, k}^{\text {calc }}\right)^{2} \\
& \text { sujeito } \quad a:\left\{\begin{array}{l}
\mathfrak{I}(m)=u \\
l_{i} \leq m_{i} \leq p_{i}, \quad i=1,2, \ldots, n
\end{array}\right.
\end{aligned}
$$

Sendo o $\Im(m)=u$ o operador matemático que determina o campo de onda $u$ em função dos vetores de parâmetros $m$ (de tamanho $n$ ) do modelo considerado. $l_{i}$ e $p_{i}$ são os vínculos associados a cada parâmetro $m_{i}$ que determinam os valores mínimos $l_{i}$ e máximos $p_{i}$ permitidos para cada parâmetro $m_{i}$.

\subsubsection{Métodos híbridos}

Cada método de otimização emprega uma estratégia de busca de uma solução cada vez melhor que venha minimizar (ou maximizar) a função objetivo tendo suas vantagens e desvantagens. Diversas estratégias híbridas de otimização podem ser construídas pela combinação de métodos com base em DFO. O intuito é desenvolver metodologias híbridas de otimização que tirem proveitos das diversas técnicas existentes. Neste trabalho, foi elaborado um algoritmo de otimização híbrido tomando como base os métodos RJT, CRS e ANMS. A técnica RJT é puramente aleatória e se presta apenas para dar o chute inicial necessário para construir a população inicial do CRS consistentes com os vínculos. Dessa população (reservatório) de modelos iniciais, é retirado, randomicamente, simplex, que será analisado pelo ANMS. A estratégia é balancear automaticamente as buscas globais e locais procuradas iterativamente pelo CRS e ANMS, respectivamente. Com isso, espera-se aumentar a eficiência geral da busca pelo ótimo global do PIS. 


\subsubsection{Técnica de salto aleatório (RJT)}

Esta técnica de otimização gera pontos (x) aleatórios no domínio de interesse no qual uma função objetivo $f(\mathbf{x})$ é avaliada em todos esses pontos. Isto gera um hyperbox, que é um subconjunto das possíveis soluções. As restrições (vínculos) impostas ao problema são constituídas pelos intervalos, $l_{i} \leq x_{i} \geq u_{i}$ (com $i=1,2, \ldots, n$ ), em que $l_{i}$ e $u_{i}$ são os limites inferior e superior do $i$-ésimo parâmetro. Assume-se que o mínimo local $x^{*}=\left[x_{1} x_{2} \ldots x_{n}\right]$ seja esperado dentro desse hyperbox. O ponto aleatório com o menor valor para a função objetivo é considerado uma aproximação para a solução ótima, $x^{*}$. O método RJT é uma técnica de otimização puramente estocástica (sem memória ou sem auto correlação), um método de Monte Carlo, que gera uma sequência aleatória de pontos dentro do hyperbox da forma:

$$
x=\left[\begin{array}{c}
x_{1} \\
x_{2} \\
\ldots \\
x_{n}
\end{array}\right]=\left[\begin{array}{c}
l_{1}+r_{1}\left(u_{1}-l_{1}\right) \\
l_{2}+r_{2}\left(u_{2}-l_{2}\right) \\
\ldots \\
l_{n}+r_{n}\left(u_{n}-l_{n}\right)
\end{array}\right]
$$

Forma esta em que $r_{i} \in\left[\begin{array}{ll}0 & 1\end{array}\right], i=1,2, \ldots, n$ são números aleatórios. O RJT é considerada uma técnica de otimização global (Bakr, 2013).

\subsubsection{Método de busca aleatória controlada (CRS)}

O CRS é uma técnica de otimização não linear baseada em DFO. Trata-se de um método de Monte Carlo modificado. Ele foi criado por Price, em 1977, ao propor um novo algoritmo de busca aleatória (Price, W. L., 1977). Ele configura-se em um método aleatório, direto e que não faz exigência acerca da diferenciação da função ou da continuidade das variáveis, além de ser aplicável na presença de vínculos. Pode ser visto também como um algoritmo populacional, em que uma população inicial de pontos, candidatos potenciais a solução do problema de otimização, é gerada aleatoriamente e contraída iterativamente em direção a um ponto em que se espera ser o ótimo global (Lima, 2006, Manzanares-Filho, N., Sousa, B. S., Camacho, R. G. R., Albuquerque, R. B. F. \& Jorge, A. B., 2007).

Para descrever o algoritmo, deve-se considerar que $M$ é o número de parâmetros do modelo (pontos) a ser determinado pela inversão sísmica. B é o espaço de parâmetros consistentes com os vínculos impostos a priori. É o interior do espaço de busca, ou seja, o domínio restrito da função objetivo, em que se encontram todas as soluções possíveis. A é um subconjunto discreto de $\mathbf{B}$ e constitui um reservatório de modelos iniciais que serão atualizados no decorrer do processo iterativo de busca pelo ótimo. $L=k(M+1)$ é o tamanho desse reservatório. Adota-se k=10. Então, 
Algoritmo CRS: para uma função $f(x): \mathfrak{R}^{M}$ a $\mathfrak{R}^{1}$, uma iteração do CRS ocorre da seguinte maneira (Lima, 2006):

1. Inicializar o valor de $L=k(M+1)$ que será o número de vetores (pontos ou modelos) de projeto de uma população em uma busca qualquer.

2. Escolher $L$ pontos (modelos) no espaço de parâmetros $\mathbf{B}$ consistentes com os vínculos e calcular o valor da função objetivo em cada ponto e armazenar na matriz A (reservatório) de dimensão $L \times(M+1)$.

3. Determinar em $\mathbf{A}$, dentre os $L$ pontos, o ponto $H$ correspondente ao maior valor da função objetivo $f(H)$, ou seja, $H$ é o pior ponto da população.

4. Escolher aleatoriamente $(M+1)$ pontos distintos $P_{1}, P_{2}, \ldots, P_{M+1}$ do conjunto de $L$ de pontos armazenados em $\mathbf{A}$. Determinar o centroide, $C$, dos $M$ pontos $P_{1}, P_{2}, \ldots, P_{M}$, a fim de determinar o próximo ponto de busca (teste), $Q$, tal que $Q=2 C-P_{M+1}$.

5. Avaliação do ponto teste $Q$.

Caso 5.1 - Se a posição do ponto teste $Q$ NÃO for consistente com os vínculos, então retorne à etapa 4 .

Caso 5.2 - Se a posição do ponto teste $Q$ for consistente com os vínculos, então prossiga.

6. Calcule $f(Q)$, o valor da função objetivo em $Q$.

7. Se $f(Q)>f(H)$. Abandonar este ponto teste e retornar para a etapa 4 .

8. Se $f(Q)<f(H)$. Neste caso o ponto teste $Q$ é incorporado (como novo ponto). Ou seja, substituir em $\mathbf{A}$ as coordenadas e o valor da função objetivo de $H$ por aquelas de $Q$.

9. Teste de convergência:

Caso 9.1 - Convergência NÃO satisfeita, então retornar-se a etapa 3 .

Caso 9.2 - Convergência satisfeita, então prossiga.

10. FIM.

Para o critério de parada pode-se usar uma das seguintes opções (Lima, 2006): 1) distribuição plana dos pontos de busca, $f(H)-f(L)<\varepsilon_{1}$ em que $f(H)$ e $f(L)$ são, respectivamente, o maior e o menor valor de função objetivo da população $(L)$ e $\varepsilon_{1}$ é o valor de tolerância adotada; 2) proximidade dos pontos de busca, $\left\|P_{i}-C\right\|<\varepsilon_{2}$, para $i=1, \ldots, L$ em que $\varepsilon_{2}$ é o valor de tolerância e $\left\|P_{i}-C\right\|$ representa a distância do $i$-ésimo ponto ao centroide 
$C$ da população $L$, dado por $C=\frac{1}{L} \sum_{i=1}^{L} P_{i}$; e 3) quando um número específico, itmax, de iterações é excedido.

Resumindo, o método CRS de Price evolui iterativamente em um esquema de atualização das variáveis de interesse de uma determinada população. É naturalmente um método de otimização global em que, a cada iteração, um único ponto teste é construído, avaliado e comparado com o pior ponto da população atual. Se for superior, o ponto teste é aceito, e o pior ponto é substituído por ele, formando uma nova população melhorada. Trata-se de um processo de busca aleatória controlada, que é repetido, até que certo critério de parada seja atingido. A solução ótima global é obtida pelo centroide da população neste momento. Portanto, é um método de busca direta global, em que pode-se identificar características de um bom algoritmo de otimização, ou seja, simplicidade conceitual, fácil implementação computacional, robustez e adequação na resolução de problemas inversos não lineares com eficiência bastante razoável.

\subsubsection{Busca simplex padrão de Nelder-Mead (SNMS)}

O algoritmo de Nelder-Mead Simplex Padrão de Nelder-Mead, Standard Nelder-Mead Simplex (SNMS), pertence a uma classe mais geral de algoritmos de pesquisa direta, e que não faz uso de derivadas, portanto trata-se uma técnica de otimização não linear, baseada em DFO. Constitui-se de um método simplex, que minimiza uma função de $M$ variáveis que, por sua vez, depende da comparação da função nos seus $(M+1)$ vértices de um simplex geral seguido pela recolocação do vértice com o valor mais alto por outro ponto de menor valor na função objetivo. O próprio simplex adapta-se à paisagem local e contrai-se a um mínimo local final. De um ponto de vista computacional, esse método mostra-se ser compacto e efetivo (Nelder, J. A. \& Mead, R., 1965). Define-se simplex $M$-dimensional como uma figura geométrica, um politopo, polígono ou hiperpoliedro, de dimensão $M$ e de volume não nulo como sendo o invólucro convexo de $M+1$ pontos independentes.

São necessários quatro coeficientes escalares para a aplicação do algoritmo de NelderMead: coeficiente de reflexão: $\rho>0$; coeficiente de expansão: $\chi>1$ com $\chi>\rho$; coeficiente de contração: $0<\gamma<1$; coeficiente de redução (shrink): $0<\sigma<1$. Na sua versão adaptada, Adaptive Nelder-Mead Simplex (ANMS), estes coeficientes dependem da dimensão $M$ do problema de otimização (Gao \& Han, 2012). Então,

$$
\rho=1,0 \chi=1+\frac{2}{M} \gamma=0,75-\frac{1}{2 M} \sigma=1-\frac{1}{M}
$$

Isso tenta superar as dificuldades de convergência encontradas pelo SNMS para problemas com altas dimensões $(M>2)$. Quando $M=2$ o ANMS torna-se o SNMS e, $\rho=1,0, \chi=2,0$, $\gamma=0,5$ e $\sigma=0,5$ (Nelder \& Mead, 1965). 
O método ANMS constitui-se em um algoritmo de busca direta que emprega um simplex no $\mathfrak{R}^{M}$, ou seja, um conjunto de $(M+1)$ pontos no espaço de $M$ dimensões em cujos vértices têm-se aproximações para o ótimo local. Então,

Algoritmo do SNMS (ANMS), sejam $x_{1}, x_{2}, \ldots, x_{M+1}$, vértices que definem um simplex no $\Re^{M}$ e $\rho, \chi, \gamma$ e $\sigma$, coeficientes de reflexão, expansão, contração e redução, respectivamente. Para uma função $f(x): \mathfrak{R}^{M}$ a $\mathfrak{R}^{1}$, uma iteração de Nelder-Mead ocorre assim (Lagarias, J. C., Reeds, J. A., Wright, M. H. \& Wright, P. E., 1998):

1. ordenação: ordenar os vértices do simplex de maneira que $f\left(x_{1}\right) \leq f\left(x_{2}\right) \leq \ldots \leq f\left(x_{n+1}\right)$. Calcular o centroide dos $n$ melhores pontos, $\bar{x}=\sum_{i=1}^{n} \frac{x_{i}}{n}$.

2. reflexão: calcular o ponto de reflexão $x_{r}=(1+\rho) \bar{x}-\rho x_{n+1}$. Se $f\left(x_{1}\right) \leq f\left(x_{r}\right)<f\left(x_{n}\right)$, aceitar o ponto $x_{r}$ e terminar a iteração.

3. expansão: se $f\left(x_{r}\right)<f\left(x_{1}\right)$, calcular o ponto de expansão $x_{e}=(1+\rho \chi) \bar{x}-\rho \chi x_{n+1}$. Se $f\left(x_{e}\right)<f\left(x_{r}\right)$, aceitar $x_{e}$ e terminar a iteração, caso contrário $\left(f\left(x_{e}\right) \geq f\left(x_{r}\right)\right)$ aceitar $x_{r}$ e terminar a iteração.

4. contração: se $f\left(x_{r}\right) \geq f\left(x_{n}\right)$ fazer uma contração:

a. contração externa: se $f\left(x_{n}\right) \leq f\left(x_{r}\right)<f\left(x_{n+1}\right)$, calcular $x_{c}=(1+\rho \gamma) \bar{x}-\rho \gamma x_{n+1}$. Se $f\left(x_{c}\right) \leq f\left(x_{r}\right)$, aceitar $x_{c}$ e terminar a iteração. Caso contrário, ir para o passo 5 .

b. contração interna: se $f\left(x_{r}\right) \geq f\left(x_{n+1}\right)$, calcular $x_{c}=(1+\gamma) \bar{x}-\gamma x_{n+1}$. Se $f\left(x_{c}\right)<f\left(x_{n+1}\right)$, aceitar $x_{c}$ e terminar a iteração. Caso contrário, ir para o passo 5 .

5. redução (shrink): calcular os vetores $v_{i}=x_{1}+\sigma\left(x_{i}-x_{1}\right), i=2, \ldots, n+1$. Os vértices (ainda fora de ordem) para a próxima iteração são $x_{i}, v_{2}, \ldots, v_{n+1}$.

Resumindo, os passos do algoritmo SNMS são: 1 . ordena decrescentemente os vértices do simplex, assim, refere-se a $x_{1}$ como o melhor vértice, e $x_{n+1}$ como o pior vértice; 2 . reflete o pior vértice em relação ao centroide; 3 . expande-se o simplex; 4. contrai o simplex refletido, reduzindo à superfície e modificando apenas o pior vértice; 5 . encolhe o simplex, reduzindo a área do simplex em torno do melhor vértice, contraindo-se os piores vértices. A Figura 5 mostra as transformações possíveis em um simplex que é atualizado em um espaço 2D via ANMS. Neste espaço, os vértices do simplex formam um triângulo. $\mathrm{O}$ triângulo formado pelos vértices $x_{l}, x_{h} \mathrm{e}$ $x_{s}$ é o simplex a ser modificado. Nesta Figura 3, $x_{l}$ é o melhor ponto, $x_{h}$ é o pior ponto (suposto ponto teste a ser substituído), $x_{s}$ é segundo pior ponto e $c$, o centroide entre $x_{l}$ e $x_{s}$. O triângulo vermelho é o simplex modificado (atualizado) após sofrer uma transformação geométrica. 


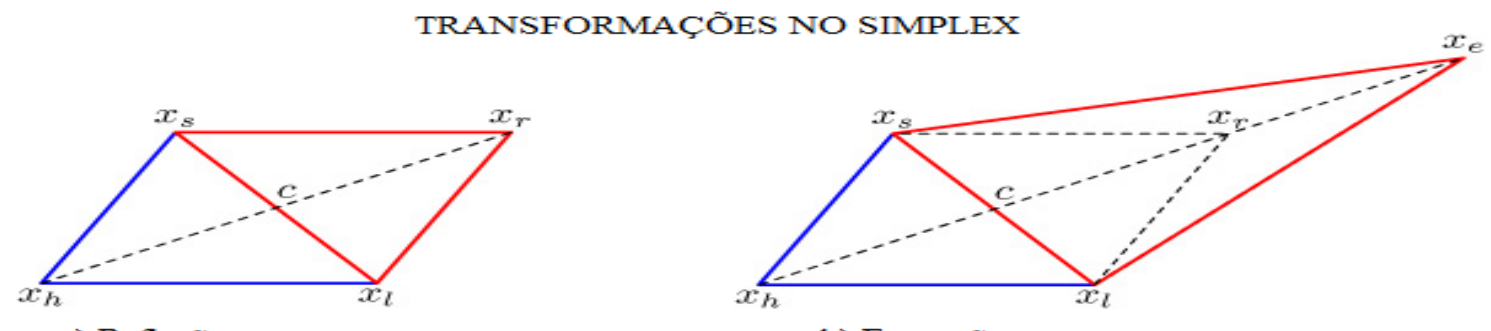

a) Reflexão

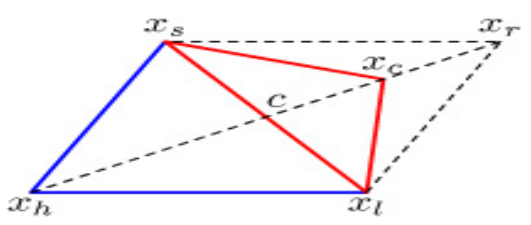

c) Contração-para fora

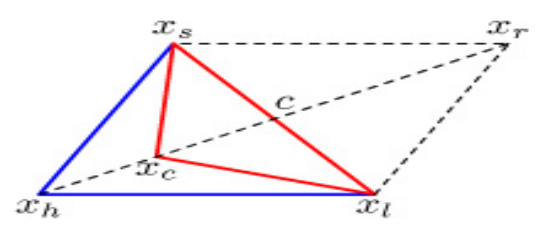

d) Contração-para dentro

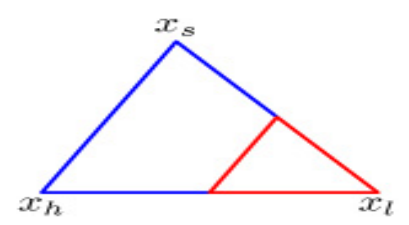

e) Redução

Figura 3: Mostra as cinco transformações no simplex 2D: (a) Reflexão $x_{e}$, (c) Contração para fora $x_{c}$, (d) Contração para dentro $x_{c}$ e (e) Redução.

Fonte: Adaptada de Lagarias, J. C., Reeds, J. A., Wright, M. H., Wright, P. E. (1998). Convergence properties of the Nelder-Mead simplex method in low dimensions. SIAM Journal on Optimization, 9(1), 112-147. doi: $10.1137 / \mathrm{s} 1052623496303470$.

\subsubsection{Critério de parada para o SNMS}

Os dois últimos critérios adotados pelo CRS podem ser adotados para o término da busca do SNMS (ANMS). O método ANMS, além de evoluir iterativamente, é definido, naturalmente, como um método de otimização local em que, a cada iteração, um único ponto teste é construído, avaliado e comparado com o pior ponto do simplex atual. Se for superior, o ponto teste é aceito, e o pior ponto é substituído por ele, formando um novo simplex, ou então o tamanho do simplex é reduzido. É um processo de busca direta, que é repetido até que certo critério de parada seja atingido. A solução ótima local é obtida pelo melhor ponto do simplex neste momento (Gao\& Han, 2012, Pedroso, L. G., 2005).

\section{METODOLOGIA}

Para resolver o problema direto sísmico, utiliza-se a equação de onda acústica sísmica em duas dimensões (2D), no domínio do tempo, para modelar o campo de propagação da onda sísmica em meios contínuos, homogêneos e isotrópicos. Isto permite calcular o sismograma sintético de reflexão para modelos de velocidades. Usa-se a técnica DFO, como método de otimização da função desajuste entre dados calculados e observados, para realizar a inversão sísmica. Para tanto, foi desenvolvido um algoritmo FWI-DFO, que resolve numericamente a equação da onda pelo FDM, e toma-se como base os métodos RJT-CRS-ANMS, para construir um algoritmo híbrido, para minimizar a função custo do Problema Inverso Sísmico (PIS). 
Portanto, foi elaborado um algoritmo híbrido de otimização ou estratégia híbrida de otimização para a modelagem FWI-DFO. A técnica DFO toma como base os métodos RJT, CRS e ANMS, ou seja, DFO=RJT-CRS-ANMS. A técnica RJT é puramente aleatória e presta-se apenas a dar o "chute" inicial necessário para construir a população inicial do CRS, o reservatório de modelos A de tamanho $L$. O reservatório $\mathbf{A}$ é um subconjunto discreto de $\mathbf{B}$, espaço de parâmetros consistentes com os vínculos. Deste reservatório de modelos iniciais, são obtidos, randomicamente, simplex(s) com $(M+1)$ vértices (modelos), que serão analisados pelo ANMS na busca do ponto ótimo local no espaço de dimensão $M$. Nesta metodologia, o ponto teste $Q$ é o ótimo alcançado pelo ANMS. Se o ponto $Q$ é tal que os critérios de convergência são satisfeitos, o processo termina e a solução ótima global é supostamente encontrada, ponto $Q$. Caso contrário, guarda-se o ponto $Q$ e seu valor $f(Q)$ em um reservatório de melhores resultados encontrados, a matriz Bsol. Em seguida, o ponto $Q$ é comparado com pior ponto $H$ da população em $\mathbf{A}$. Se o ponto $Q$ for melhor que o ponto $H$, substitui-se este por aquele. Se não, verifica-se se foi atingido o número de visitas máximo permitido ao reservatório $\mathbf{A}$. Se sim, a solução ótima é a melhor dentre as armazenadas em Bsol. Se não, retorna-se ao início do processo. A estratégia é balancear, automaticamente, as buscas globais e locais procuradas iterativamente pelo CRS e ANMS, respectivamente. Com isso, espera-se aumentar a eficiência da busca pelo ótimo global. Nesta abordagem, a convergência dá-se pelo valor da função objetivo, ou seja, $f(x)<\varepsilon$. Na qual, $\varepsilon$ é valor da tolerância preestabelecido. O processo termina quando se atinge essa convergência ou quando se ultrapassa o número de vistas permitidas ao reservatório A. A descrição do algoritmo FWI-DFO mostra-se a seguir:

\section{Algoritmo FWI-DFO=RJT-CRS-ANMS:}

1. inicializar o valor da população $L=k(M+1)$ e do número máximo de visitas permitidas ao reservatório $\mathbf{A}$;

2. aplicar a técnica RJT para gerar os $L$ pontos (modelos iniciais) no espaço de parâmetros B consistentes com os vínculos e calcular o valor da função objetivo em cada ponto e armazenar na matriz A (reservatório) de dimensão $L \times(M+1)$;

3. determinar em $\mathbf{A}$, dentre os $L$ pontos, o ponto $H$ correspondente ao maior valor da função objetivo $f(H)$, ou seja, $H$ é o pior ponto da população.

4. gerar um simplex, escolhendo aleatoriamente $(M+1)$ pontos distintos $P_{1}, P_{2}, \ldots, P_{M+1}$ do conjunto de $L$ de pontos armazenados em A. Ordenar, crescentemente, estes pontos. Identificar o polo $P_{M+1}$ (pior vértice). Determinar o centroide, $C$, dos $M$ pontos $P_{1}, P_{2}, \ldots, P_{M}$;

5. aplicar o ANMS para realizar as operações de reflexão, expansão, contração ou a redução no simplex para obter o ponto ótimo, o ponto teste $Q$ e $f(Q)$ nesta etapa;

6. teste de convergência para a tolerância para função objetivo $f(x)$ no ponto teste: 
Caso 6.1 - Convergência NÃO satisfeita, $f(Q)>\varepsilon$, então:

- $\quad$ neste caso, o ponto teste $Q$ é incorporado (como novo ponto). Ou seja, acrescentar em Bsol , as coordenadas de $Q$ e o valor da função objetivo $f(Q)$;

- $\quad$ avaliação do ponto teste $Q$

1. Se $f(Q)>f(H)$, ir para etapa 7 .

2. Se $f(Q)<f(H)$. Neste caso, o ponto teste $Q$ é incorporado (como novo ponto). Ou seja, Substituir em $\mathbf{A}$, as coordenadas e o valor da função objetivo de $H$ por aquelas de $Q$, ir para etapa 7.

Caso 6.2 - Convergência satisfeita, $f(Q)<\varepsilon$, então ir para etapa $\mathbf{8}$.

7. Teste de convergência das visitas ao reservatório $\mathbf{A}$ :

Caso 7.1 - Convergência NÃO satisfeita, então retornar-se a etapa 3.

Caso 7.2 - Convergência satisfeita, então prossiga.

\section{FIM}

O algoritmo FWI-DFO tenta atingir o critério de convergência pelo ANMS, que constitui um processo de busca por um simplex obtido do subespaço discreto de conjuntos de modelos que é contraído, pelo o CRS, a cada visita ao reservatório A. Espera-se, portanto, que este procedimento procure escapar de mínimos locais. Resumindo, o CRS contrai o conjunto de $L$ modelos, um grande simplex não convexo, em direção ao seu ótimo global. Enquanto que o ANMS contrai um simplex de tamanho $(M+1)$, subtraído daquele espaço cada vez mais reduzido, para encontrar seu ótimo local. $\mathrm{O}$ que se espera ser o ótimo global, que poderia ser encontrado pelo CRS, caso ele evoluísse de maneira tradicional. Vale ressaltar, novamente, que, neste algoritmo, híbrido de otimização, a estratégia é balancear automaticamente as buscas globais e locais procuradas iterativamente pelo CRS e ANMS, respectivamente.

\subsection{Modelagem sísmica 2D}

Nesta pesquisa foi utilizado um modelo base cuja geometria representa as camadas do subsolo, em uma aproximação que corresponde a uma seção plana de dimensões unitárias: $L_{m} \times H_{m}=1,0 \times 1,0$. Trata-se de região plana, mas com velocidades diferentes. Este modelo denomina-se modelo de velocidades de camadas e posição do refletor. $\mathrm{O}$ experimento sísmico sintético foi idealizado por uma fonte sísmica, simulada pela wavelet de Ricker, com frequência de pico $v_{0}=10 \mathrm{~Hz}$, localizada a meia distância do plano da superfície e próxima à superfície dos modelos ou a 5 pontos de malha a partir do primeiro elemento da borda superior. Foi utilizada uma linha de receptores (geofones) localizados em uma determinada profundidade, ou a 7 pontos de malha a partir da fonte para gravar os dados sísmicos sintéticos que serão utilizados no problema inverso - ver Figura 4 (a). No processo de discretização do domínio espacial pelo FDM, 
usou-se uma malha com $141 \times 141$ pontos nodais com espaçamento $h=\Delta x=\Delta y=0,0071$ e um tempo de propagação da onda, janela de tempo, de $t=1,5 \mathrm{~s}$ com tempo de amostragem, passo, $\Delta t=7,5 \mathrm{~ms}$. Para avaliar o critério de parada da otimização, adotou-se um valor para a tolerância de $\varepsilon=10^{-3}$ para o ANMS e número máximo de visitas ao reservatório $\mathbf{A}$, igual a 20 para o CRS. Além disso, foi estabelecido um limite máximo de 100 avaliações da função objetivo para que o ANMS tentasse convergir para cada visita ao reservatório $\mathbf{A}$.

Utiliza-se um conjunto misto de parâmetros para caracterizar os modelos desenvolvidos, os quais constituem-se de velocidades das camadas e da posição dos refletores $\left[v_{1} \ldots v_{p} h_{1} \ldots h_{q}\right]$. Os vínculos associados a este conjunto de parâmetros são representados pelos limites mínimos e máximos permitidos para seus valores, de acordo com as restrições impostas por cada modelo considerado nesta pesquisa, na qual se desenvolvem cinco modelos para a aplicação da metodologia FWI-DFO, seguindo essa linha básica de construção. Os modelos são gerados em uma sequência crescente, em complexidades que envolvem refração, reflexão, difração e espalhamento, como no exemplo a seguir.

\subsubsection{Modelo de velocidades de camadas horizontais [V]}

O primeiro modelo [V] (mais simples) apresenta três camadas, cada qual com suas respectivas velocidades desconhecida $V_{1}, V_{2}$ e $V_{3}$. A inversão sísmica irá encontrar apenas o valor dessas três velocidades. Neste caso, as posições dos refletores são conhecidas a priori e os parâmetros do modelo são do mesmo tipo (velocidades). O vetor de parâmetros do modelo [V] é $\left[\begin{array}{lll}V_{1} & V_{2} & V_{3}\end{array}\right]$ - ver Figura 4 (b).
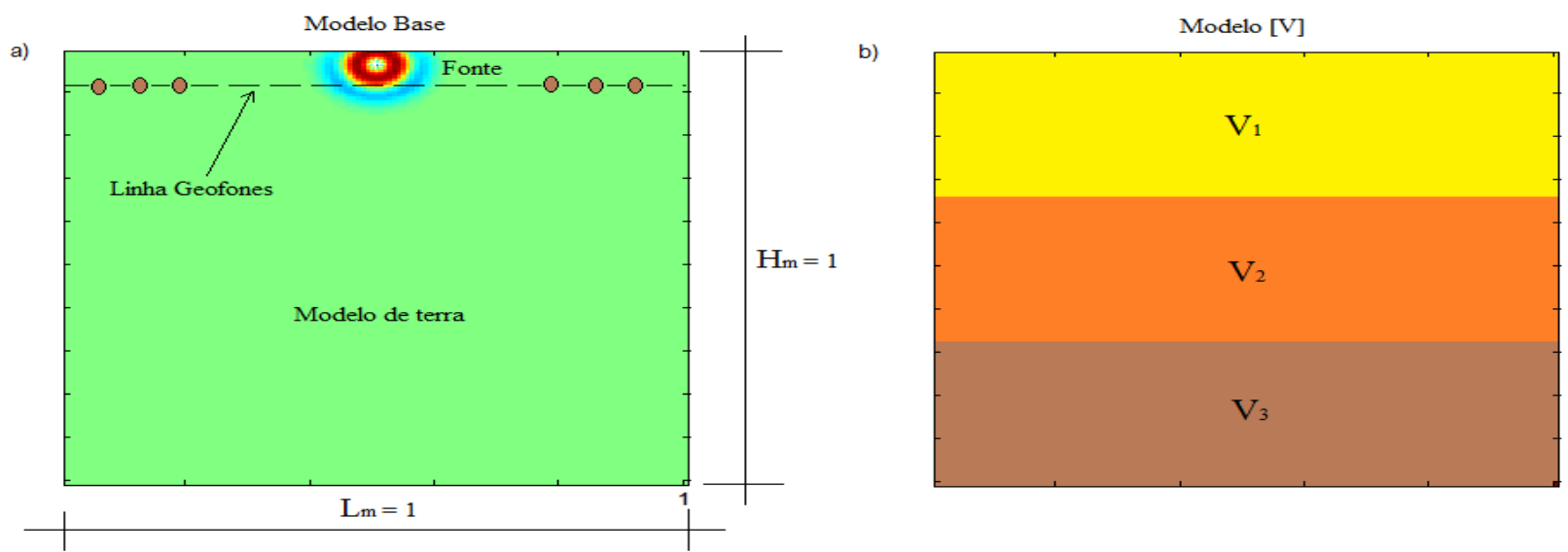

Figura 4: (a) Geometria do modelo base de camadas; (b) Modelo de velocidades de camadas horizontais, Modelo [V]. 
Com base neste modelo simples, gera-se mais quatro outros modelos de maior complexidade. Então, tem-se o modelo de velocidades de camadas e posição dos refletores, modelo [VH]. Este segundo modelo possui três camadas. Entretanto, como não se conhece a posição dessas camadas, foram acrescentados mais dois parâmetros, a saber: as posições da primeira e da segunda camada. Consequentemente, obtem-se, além das velocidades $V_{1}, V_{2}$ e $V_{3}$, as posições dos refletores $h_{1}$ e $h_{2}$. O vetor de parâmetros do modelo [VH] é $\left[V_{1} V_{2} V_{3} h_{1} h_{2}\right.$ ]; modelo de velocidades de camadas e posição dos refletores inclinados, modelo [VHI]. Este terceiro modelo aborda a situação de um refletor inclinado. Neste modelo, encontra-se as velocidades das duas camadas e a posição do respectivo refletor inclinado. O vetor de parâmetros do modelo [VHI] é $\left[V_{1} V_{2} h_{1} h_{2}\right]$; modelo de velocidades de camadas com difração, modelo [VHD]. Este quarto modelo considera o efeito de difração. Em tal modelo, existe um ponto de difração cuja localização não se conhece. O objetivo é encontrar a posição desse ponto de difração, e as velocidades das duas camadas. A posição do ponto de difração distingue uma camada da outra. $O$ vetor de parâmetros do modelo [VHD] é $\left[V_{1} V_{2} h_{1} h_{2}\right]$; Modelo de velocidades de camadas com domo de Sal [VHS]. Finalmente, esse quinto modelo analisa o efeito de uma região refletora. $O$ refletor em questão é um domo de sal de geometria circular. Neste caso, encontra-se a posição desse refletor, o centro do domo de sal, e as velocidades das camadas. O vetor de parâmetros do modelo [VHS] é $\left[V_{1} V_{2} h_{1} h_{2}\right]$. A Figura 5 mostra em detalhes esses últimos quatros modelos.

a) Modelo [VH]

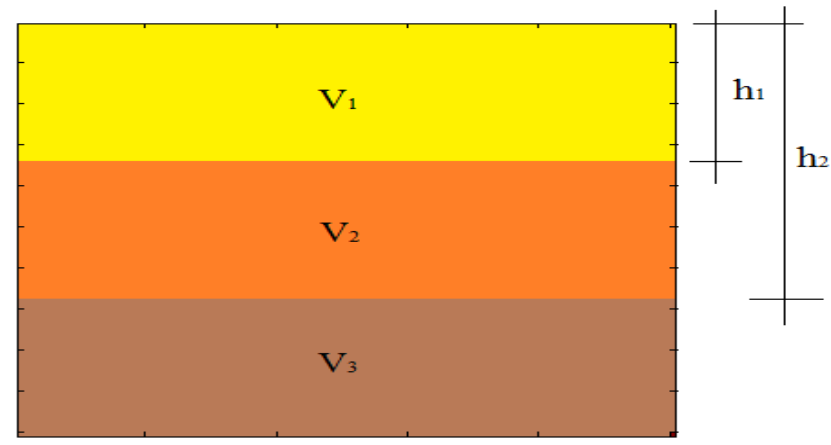

c) Modelo [VHDI

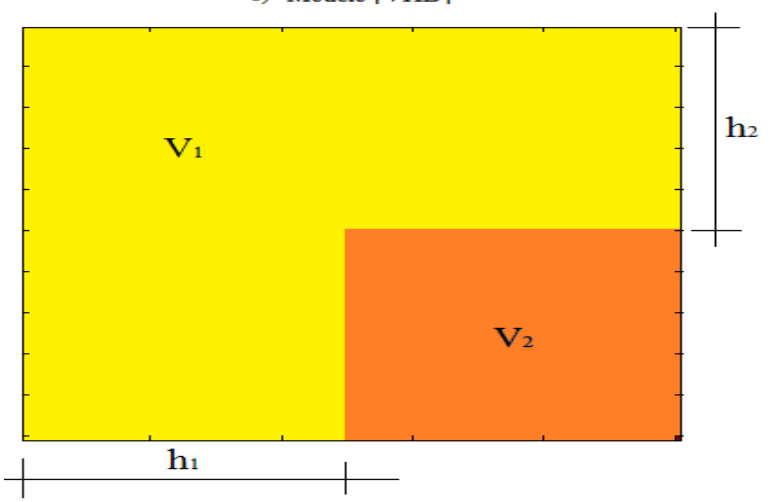

b) Modelo [VHI]

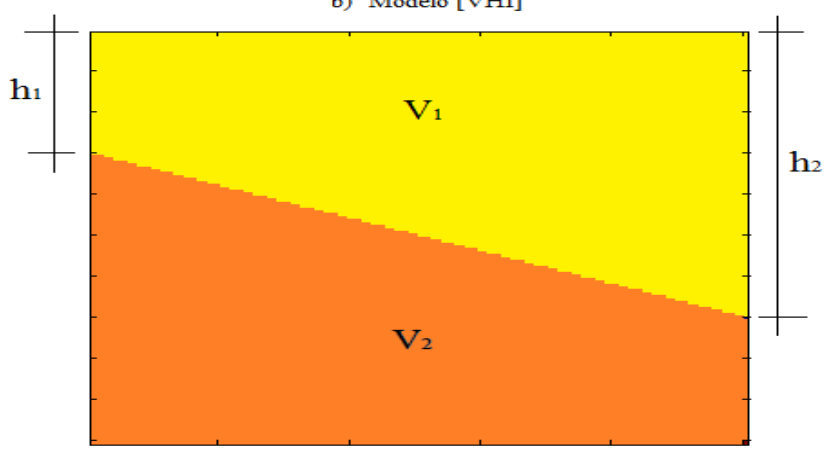

d) Modelo [VHS]

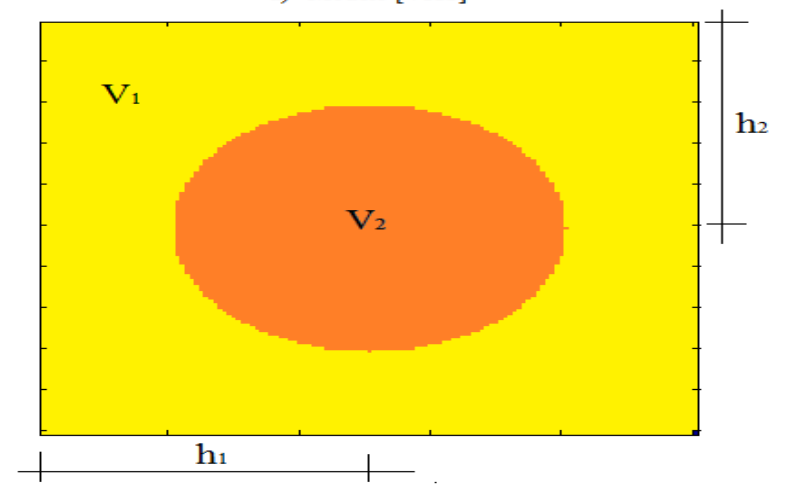

Figura 5: Modelos de velocidades: (a) [VH], (b) [VHI], (c) [VHD] e (d) [VHS], respectivamente. 
Os parâmetros $\left(V_{1}, \ldots, V_{p}, h_{1}, \ldots, h_{q}\right)$, para cada modelo, estão relacionados de acordo com os dados da Tabela 1 a seguir:

Tabela 1:

Os modelos e seus respectivos parâmetros reais.

\begin{tabular}{|c|c|c|c|c|c|}
\hline $\begin{array}{l}\text { Parâmetro } \\
\text { Modelo }\end{array}$ & $\mathbf{V}_{1}$ & $\mathbf{V}_{2}$ & $\mathbf{V}_{3}$ & $\mathbf{h}_{1}$ & $\mathbf{h}_{2}$ \\
\hline $\mathrm{V}$ & 1 & 2 & 4 & --- & --- \\
\hline $\mathrm{VH}$ & 1 & 2 & 4 & 0,3333 & 0,6667 \\
\hline VHI & 2 & 4 & --- & 0,3000 & 0,7000 \\
\hline VHD & 2 & 4 & --- & 0,5000 & 0,5000 \\
\hline VHS & 2 & 4 & --- & 0,5000 & 0,5000 \\
\hline
\end{tabular}

Para cada modelo, estabelecem-se limites inferior e superior para os respectivos parâmetros. Isso caracteriza o processo de otimização baseado em DFO com restrição, ou seja, otimização não linear com vínculos. Estes limites estão apresentados na Tabela 2 para todos os modelos.

Tabela 2:

Os limites inferior $\left(L_{\text {inf }}\right)$ e superior $\left(L_{\text {sup }}\right)$, vínculos.

\begin{tabular}{c|cc|cc|cc|cc|cc}
\hline Par./Lim. & \multicolumn{2}{|c|}{$\mathbf{V}_{\mathbf{1}}$} & \multicolumn{2}{|c|}{$\mathbf{V}_{\mathbf{2}}$} & \multicolumn{2}{c|}{$\mathbf{V}_{\mathbf{3}}$} & \multicolumn{2}{c|}{$\mathbf{1 0 h}_{\mathbf{1}}$} & \multicolumn{2}{c}{$\mathbf{1 0 h}_{\mathbf{2}}$} \\
Modelo & $\mathbf{L}_{\text {inf }}$ & $\mathbf{L}_{\text {sup }}$ & $\mathbf{L}_{\text {inf }}$ & $\mathbf{L}_{\text {sup }}$ & $\mathbf{L}_{\text {inf }}$ & $\mathbf{L}_{\text {sup }}$ & $\mathbf{L}_{\text {inf }}$ & $\mathbf{L}_{\text {sup }}$ & $\mathbf{L}_{\text {inf }}$ & $\mathbf{L}_{\text {sup }}$ \\
\hline V & 0,50 & 1,50 & 1,50 & 2,50 & 3,00 & 5,00 & --- & -- \\
VH & 0,50 & 1,50 & 1,50 & 2,50 & 3,00 & 5,00 & 1,00 & 5,00 & 5,00 & 9,00 \\
VHI & 1,00 & 3,00 & 3,00 & 5,00 & --- & 1,00 & 5,00 & 5,00 & 9,00 \\
VHD & 1,00 & 3,00 & 3,00 & 5,00 & --- & 2,00 & 8,00 & 2,00 & 8,00 \\
VHS & 1,00 & 3,00 & 3,00 & 5,00 & --- & 2,20 & 7,80 & 2,20 & 7,80 \\
\hline
\end{tabular}

\section{RESULTADOS E DISCUSSÕES}

Nesta seção, são mostrados os resultados obtidos pelas simulações geradas pelo FWI-DFO aplicado aos cinco modelos estudados. Primeiramente, detalha-se o modelo [V]. Os dados para este modelo estão apresentados na Tabela 1 e na Tabela 2. Os valores para os parâmetros $V_{1}, V_{2} \mathrm{e}$ $V_{3}$, observados e calculados (ótimo global) para o modelo $[\mathrm{V}]$, estão relacionados de acordo com os dados da Tabela 3, assim como o valor alcançado pela função objetivo e o tempo de processamento em um core $i 5$.

Tabela 3:

Resultados e comparação para o modelo [V].

\begin{tabular}{c|c|c|c|c|c}
\hline Parâmetros & $\mathbf{V}_{\mathbf{1}}$ & $\mathbf{V}_{\mathbf{2}}$ & $\mathbf{V}_{\mathbf{3}}$ & $\begin{array}{c}\text { Função } \\
\text { Custo }\end{array}$ & $\begin{array}{c}\text { Tempo } \\
\text { (S) }\end{array}$ \\
\hline Valores & 1 & 2 & 4 & & \\
Ótimo & 1,0000 & 2,0000 & 3,9985 & 0,0047 & 153,74 \\
\hline
\end{tabular}


A Figura 6 (a) mostra o reservatório de modelos em seus estágios inicial, $\mathbf{A I}$ e final, $\mathbf{A F}$. Este reservatório tem 32 modelos identificados pela ordem em que foram gerados aleatoriamente com seus respectivos valores da função objetivo $\phi(\mathbf{m})$. A atualização do reservatório ocorre de tal modo que os modelos com valores mais altos para $\phi(\mathbf{m})$, discos vermelhos, sejam substituídos por outros de menor valor, discos azuis, à medida que o processo de busca da solução ótima evolui.
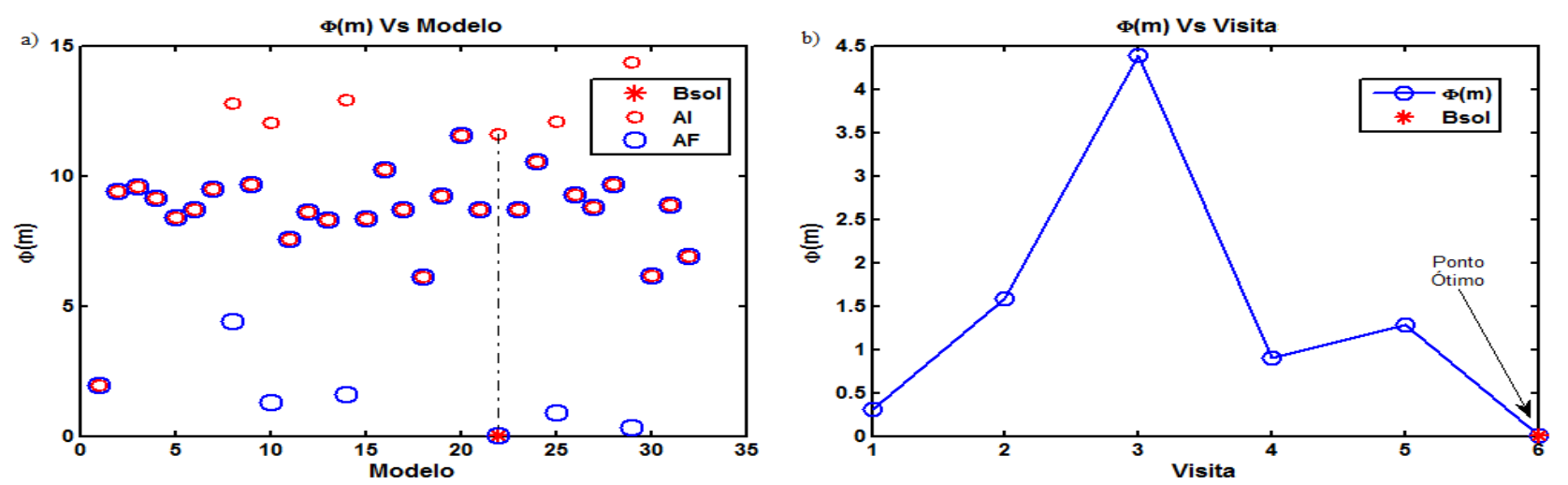

Figura 6: (a) Reservatório de modelos inicial (AI) e final (AF), e solução ótima (Bsol); (b) Valores da função objetivo $\phi(\mathrm{m})$ versus visita ao reservatório de modelos AI para o modelo [V].

Essa Figura 6 mostra que apenas seis modelos (os piores) foram substituídos, e que o modelo de número 22 foi o último a ser substituído, agora, pelo ponto ótimo. Neste momento, o critério de convergência, adotado pelo ANMS, foi atingido, terminando o processo de busca pelo seu ótimo local. Este ponto em AF é considerado o ótimo global procurado pelo CRS completando, assim, o algoritmo FWI-DFO. A Figura 6 (b) mostra os valores de $\phi(\mathbf{m})$ para os seis modelos [V] otimizados correspondentes à visita ao reservatório de modelos $\mathbf{A}$ corrente.

A Figura 7 (a) mostra, no espaço de parâmetros $V_{1}, V_{2}$ e $V_{3}$, o reservatório de modelos, com seus 32 modelos, em seu estágio inicial, AI (bolas vermelhas) e final, AF (bolas azuis).
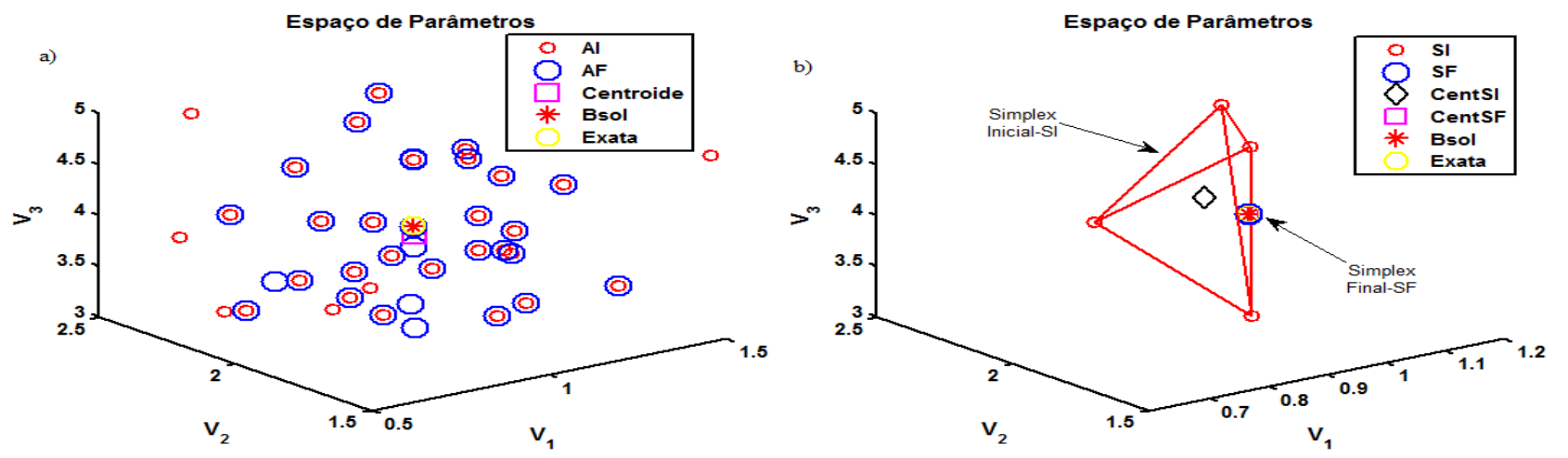

Figura 7:

(a) Reservatório de modelos inicial AI e final AF ; (b) Contração do simplex na visita de $\mathbf{n}^{\circ} 6$ para o modelo [V]. 
Dentre esses seis modelos, as bolas vermelhas isoladas foram substituídas por outras com melhores resultados, as bolas azuis isoladas. Atualizando, assim, o reservatório. Nessa Figura 7 mostra o centroide da população de AF (quadrado em cor magenta), a melhor solução alcançada pelo ANMS (asterisco vermelho), que ocorreu na visita de número seis, após 44 iterações. A Figura 7 (a) também mostra a solução exata (bola amarela). É possível observar que essas duas últimas quantidades superpõem-se em valores. A Figura 7 (b) mostra, no estágio inicial SI (bolas vermelhas) e final SF (bolas azuis), o simplex explorado pelo ANMS, na visita de número seis, visita ótima. Vê-se também, o centroide do SI (diamante preto) e o centroide do SF (quadrado magenta). Tem-se também a melhor solução alcançada pelo ANMS (asterisco vermelho) e a solução exata (bola amarela). As três últimas quantidades superpõem-se em valores. Pode-se ver também o decaimento do tamanho do simplex. O tamanho do SI é 1,65 e do SF é 0,01. Isto mostra a redução do simplex ao ponto de ótimo local pelo ANMS. Pode-se observar que a solução ótima encontrada coincide praticamente com o valor exato, neste caso.

A Figura 8 (a) mostra os resultados encontrados para todas as visitas ao reservatório $\mathbf{A}$ em comparação com seus respectivos valores reais (linha preta). Nessa Figura, o modelo é identificado por uma linha vertical traçada a partir de cada visita. Isto permite identificar os parâmetros de modelos $V_{1}, V_{2}$ e $V_{3}$ (discos azul, verde e magenta) para as respectivas velocidades das camadas do modelo geológico [V] que correspondem ao ponto de ótimo local alcançado pelo ANMS. Vê-se a identificação do ponto ótimo (asterisco vermelho), bem como a visita correspondente ao ponto ótimo. Considera-se o ótimo global como sendo o melhor dentre estes ótimos locais encontrados pelo ANMS. Para o modelo [V], o ótimo global foi alcançado na visita de número 6 para um total de 20 visitas permitidas. Nesta visita, foi alcançado o critério de parada exigido pelo método ANMS, finalizando a busca pelo ponto ótimo global.
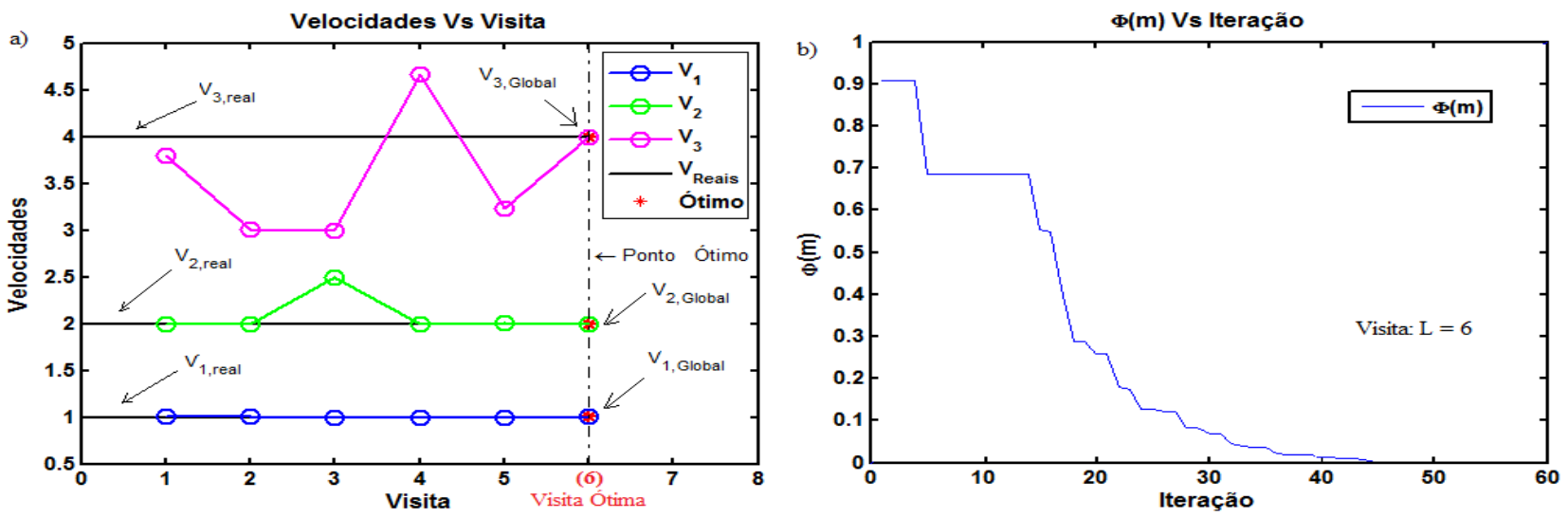

Figura 8: (a) Evolução dos valores dos parâmetros $V_{1}, V_{2}$ e $V_{3}$; (b) Valores da função objetivo $\phi(\mathbf{m})$ versus visita ao reservatório A para o modelo [V].

Especialmente neste caso, o mínimo local alcançado pelo método ANMS corresponderia exatamente ao mínimo global do problema alcançado pelo método CRS original, ou, se o número de visitas permitido, em algoritmo eleito, fosse suficientemente elevado para atingi-lo. Nesta 
situação extrema, as duas metodologias equivalem-se sob o ponto de vista de resultados. Também, pode-se observar que, quanto mais distante a camada da fonte sísmica, mais dificuldade o algoritmo tem de convergir para o valor do parâmetro correspondente (neste caso, $V_{3}$ ). Isso poderá estar relacionado ao fato da onda chegar com menor intensidade nas camadas mais afastadas. A Figura 8 (b) mostra o gráfico da função desajuste (erro) $\phi(\mathbf{m})$ com o passar das iterações pelo ANMS na visita de número seis $(L=6)$ para o modelo [V]. O processo termina na iteração de número 44 com um erro de 0,0047 .

Para o modelo [V], a Figura 9 (a) mostra as soluções ótimas locais encontradas pelo ANMS para as seis visitas consecutivas $\left(L_{1}, L_{2}, L_{3}, L_{4}, L_{5}\right.$ e $\left.L_{6}\right)$ ao reservatório de conjuntos de parâmetros de modelos $\mathbf{A}$. Pode-se observar que algumas diferem pouco da solução real, enquanto outras diferem um tanto mais. Isso é uma característica do ANMS. Ele pode ter ficado aprisionado em um mínimo local, ou o simplex planificou ou ficou em forma de agulha. Essas duas últimas situações não levam aos mínimos locais esperados, e sim a resultados ruins. A Figura 9 (b) mostra a saída dos resultados pelo algoritmo FWI-DFO, em que se compara o modelo real com o calculado pela inversão sísmica. Eles mostram-se praticamente iguais.
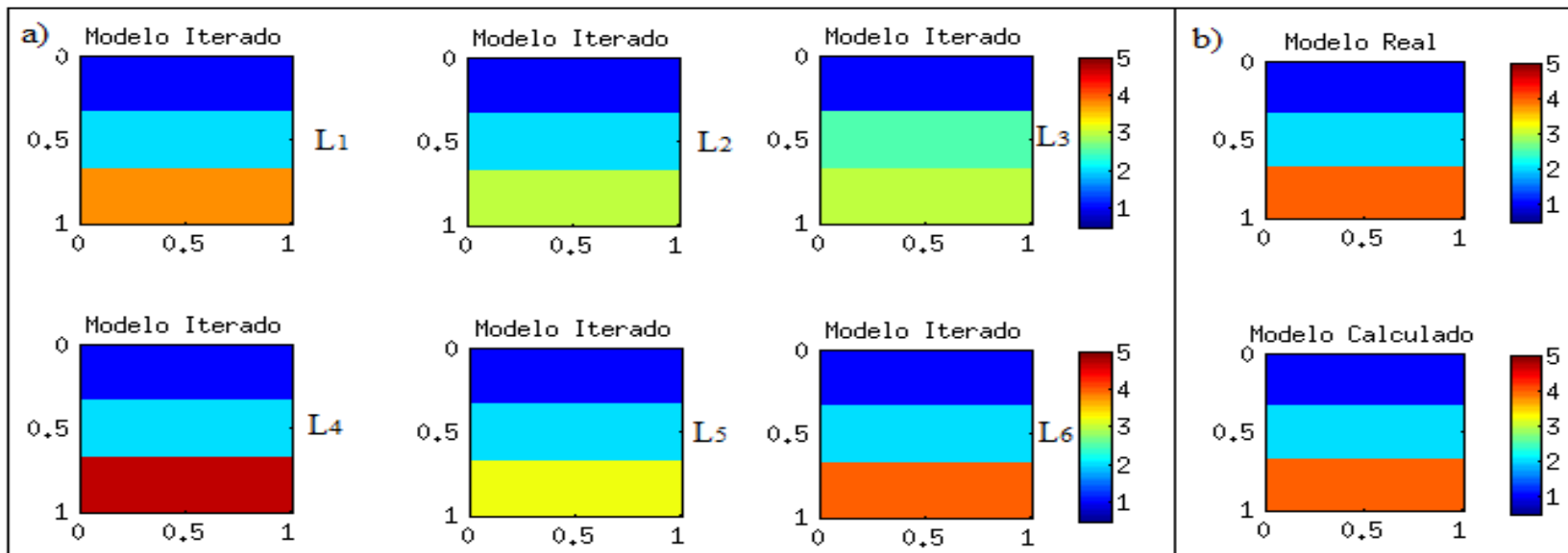

Figura 9: (a) Evolução dos pontos ótimos locais para as seis visitas ao reservatório $\mathbf{A}\left(L_{1}, L_{2}, L_{3}, L_{4}, L_{5}\right.$ e $\left.L_{6}\right)$ pelo ANMS; (b) Comparação do modelo real e calculado para o modelo [V].

A Figura 10 (a) e (b) mostra instantâneos da propagação do campo de ondas nas camadas do modelo geológico [V] referente à simulação sísmica, $V=[1,00002,00003,9985]$, para as janelas de tempo $t=0,54 \mathrm{~s}$ e $t=0,65 \mathrm{~s}$, respectivamente. Pode-se identificar a posição da fonte (asterisco branco) a cinco pontos de malha da superfície, e a linha de geofones (linha preta pontilhada) a doze pontos de malha da superfície. As três camadas com velocidades $V_{1}=1$ (azul escuro), $V_{2}=2$ (azul claro) e $V_{3}=3,9985$ (vermelho). As camadas estão dividas pelos refletores $1 \mathrm{e}$ 2 . Observam-se os efeitos de reflexão e de refração da onda acústica nas interfaces (refletores) entre camadas. Na Figura 10 (a), se observa apenas estes efeitos no refletor 1 porque o tempo de propagação não foi suficiente para que a frente de onda chegasse ao refletor 2 . Enquanto que, na 
Figura 10 (b), se observam esses efeitos nos dois refletores. Observa-se também a simetria dos gráficos em relação a uma reta vertical que passa pela fonte. Isso é um forte indício de veracidade do modelador de onda utilizado. Os valores das velocidades das camadas serviram como suporte para as intensidades, normalizadas, do campo de onda. As Figuras 10 (c) e 10 (d) são representações 3D desses casos, respectivamente. Sendo, dessa vez, com as intensidades reais para o campo de onda.
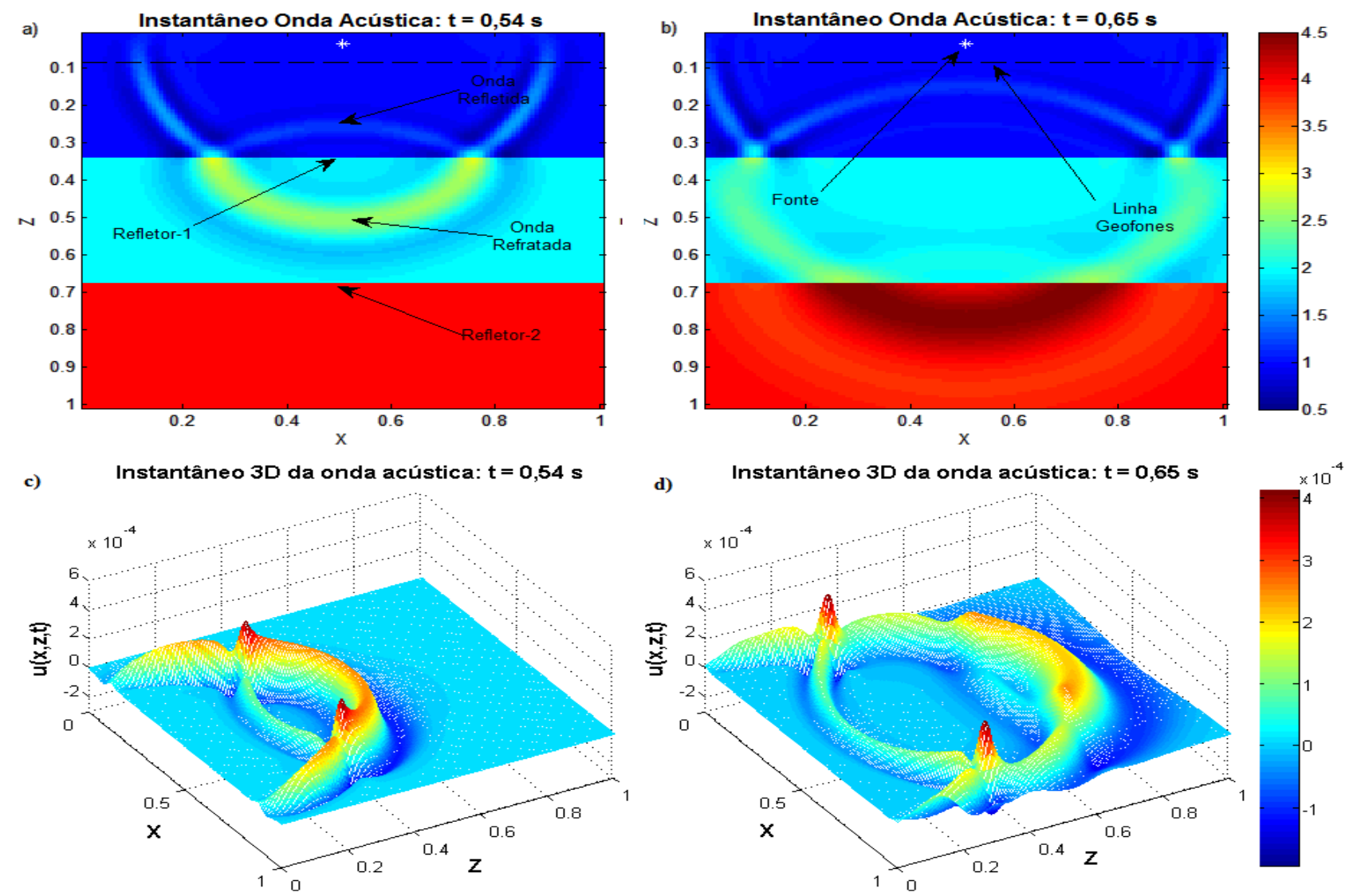

Figura 10: a) e b) São instantâneos da propagação do campo de ondas nas três camadas do modelo [V] para a janela de tempo $t=0,54 \mathrm{~s}$ e $t=0,65 \mathrm{~s}$, respectivamente; c) e d) São representações 3D desses casos.

A Figura 11 (a) e (c) mostram os Sismogramas dos modelos, $V=[1,0000$ 2,0000 3,9985] e $V=[1,00001,99863,7986]$, correspondentes aos dois melhores valores da função objetivo, $\phi=0,0047$ e $\phi=0,3120$, encontrados nas simulações. Nesses, identificam-se o registro da onda direta (gráfico triangular) e as reflexões (parábolas) ocorridas nas interfaces entre as camadas 1 e 2 , refletor 1 . E, entre as camadas 2 e 3 , refletor 2 . As bordas são consideradas não reflexivas, mas deixam algum resíduo. A Figura 11 (b) e (d) mostram os traços registrados no geofone central ( 71 pontos de malha) para os dois casos anteriores. 

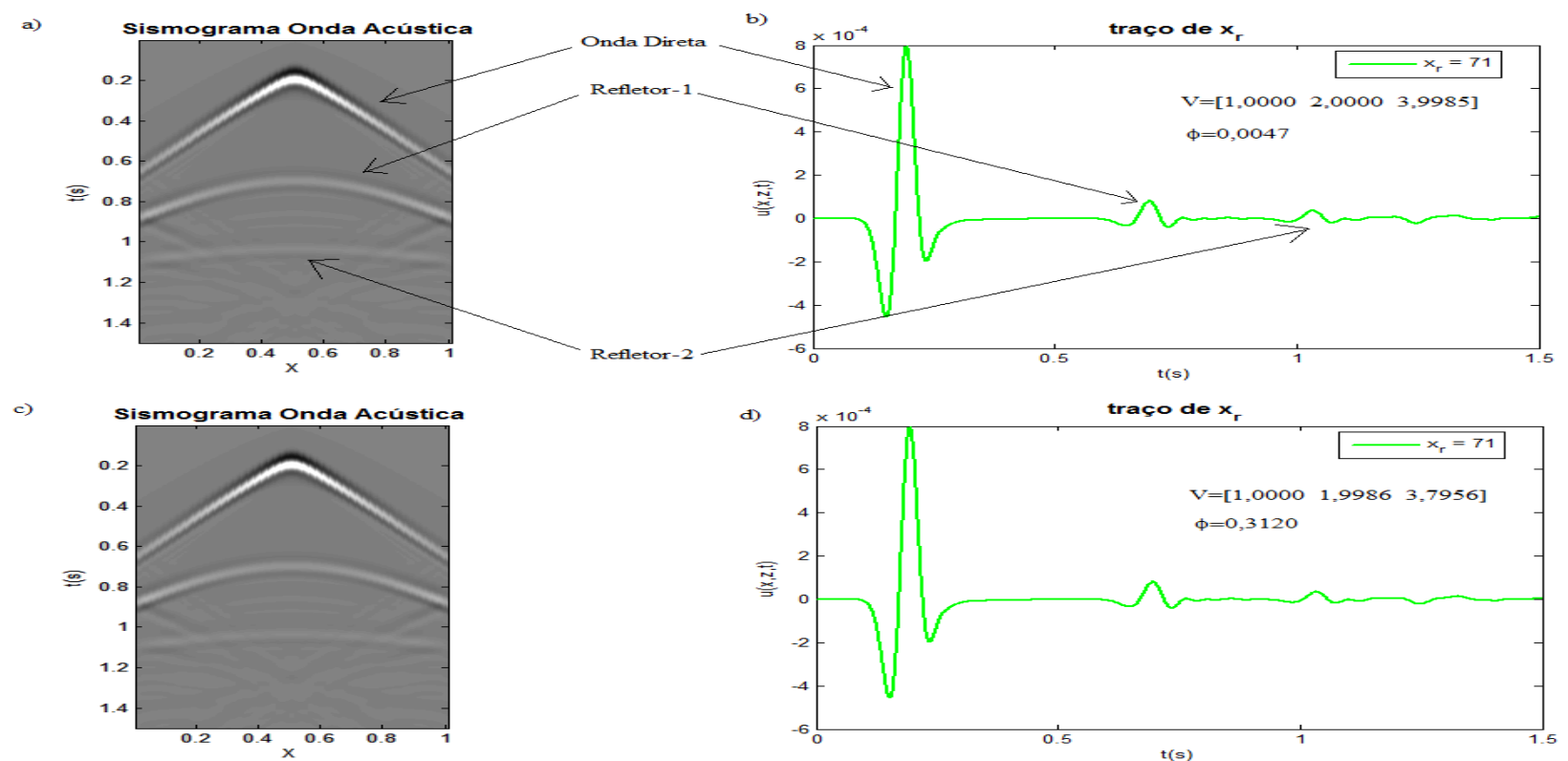

Figura 11: (a) e (c) Sismogramas para os dois melhores valores da função custo $\phi(m)$; (b) e (d) Traços na posição central do sismograma para estes valores para o modelo [V].

Nelas, é possível identificar a assinatura da onda direta e as das reflexões como antes. Pode-se ver que os modelos e suas assinaturas gráficas são bastante próximos, mas os correspondentes valores para a função objetivo são bastante diferentes. Isso mostra que a função objetivo mostrou-se ser bastante sensível a pequenas alterações nos parâmetros de modelos que constituem os modelos geológicos.

A partir daqui, detalha-se alguns resultados para o modelo [VHS]. As considerações são análogas às referenciadas ao modelo [V]. Os dados para este modelo estão apresentados na Tabela 4. Os parâmetros para este modelo são $V_{1}, V_{2}, h_{1}$ e $h_{2}$. Os valores para os $h(s)$ foram multiplicados por 10 para ficarem na mesma ordem de grandeza dos $v(s)$.
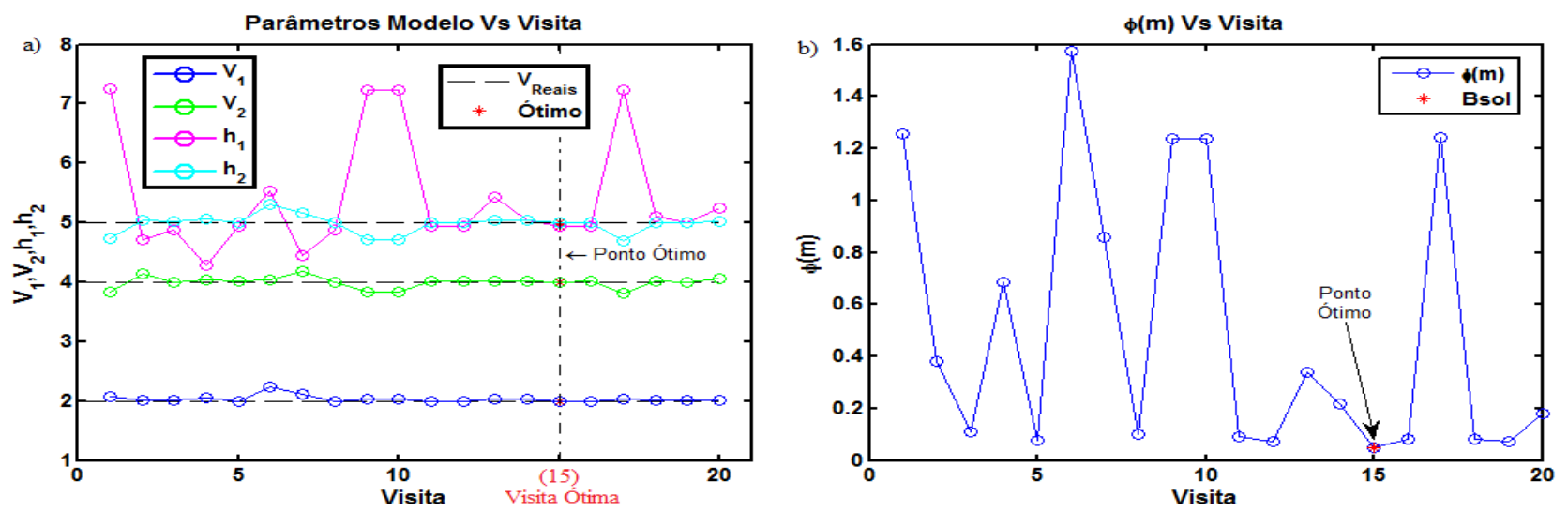

Figura 12: (a) Evolução dos valores dos parâmetros $V_{1}, V_{2}, h_{1}$ e $h_{2}$; (b) Valores da função objetivo $\phi(\mathbf{m})$ versus visita ao reservatório $A$ para o modelo [VHS]. 
A Figura 12 (a) mostra os resultados encontrados pela simulação para os modelos e seus respectivos parâmetros correspondentes à inversão sísmica para todas as visitas ao reservatório A em comparação com seus respectivos valores reais (linha preta).

Nessa Figura, o modelo é identificado por uma linha vertical traçada a partir de cada visita. Isso permite identificar os parâmetros $V_{1}, V_{2}, h_{1}$ e $h_{2}$, discos azul, verde, magenta e cianeto, respectivamente, para as camadas deste modelo, que correspondem ao ponto de ótimo local alcançado pelo ANMS para cada visita. O ponto ótimo (asterisco vermelho) é identificado, bem como identifica-se a visita correspondente a ele.

Para o modelo [VHS], a Figura 13 (a) mostra as soluções ótimas locais encontradas pelo ANMS para algumas visitas $\left(L_{1}, L_{3}, L_{6}, L_{10}, L_{15}\right.$ e $\left.L_{20}\right)$ ao reservatório de conjuntos de parâmetros A . A Figura 13 (b) mostra a saída dos resultados pelo algoritmo FWI-DFO, em que se compara o modelo real com o calculado pela inversão sísmica.

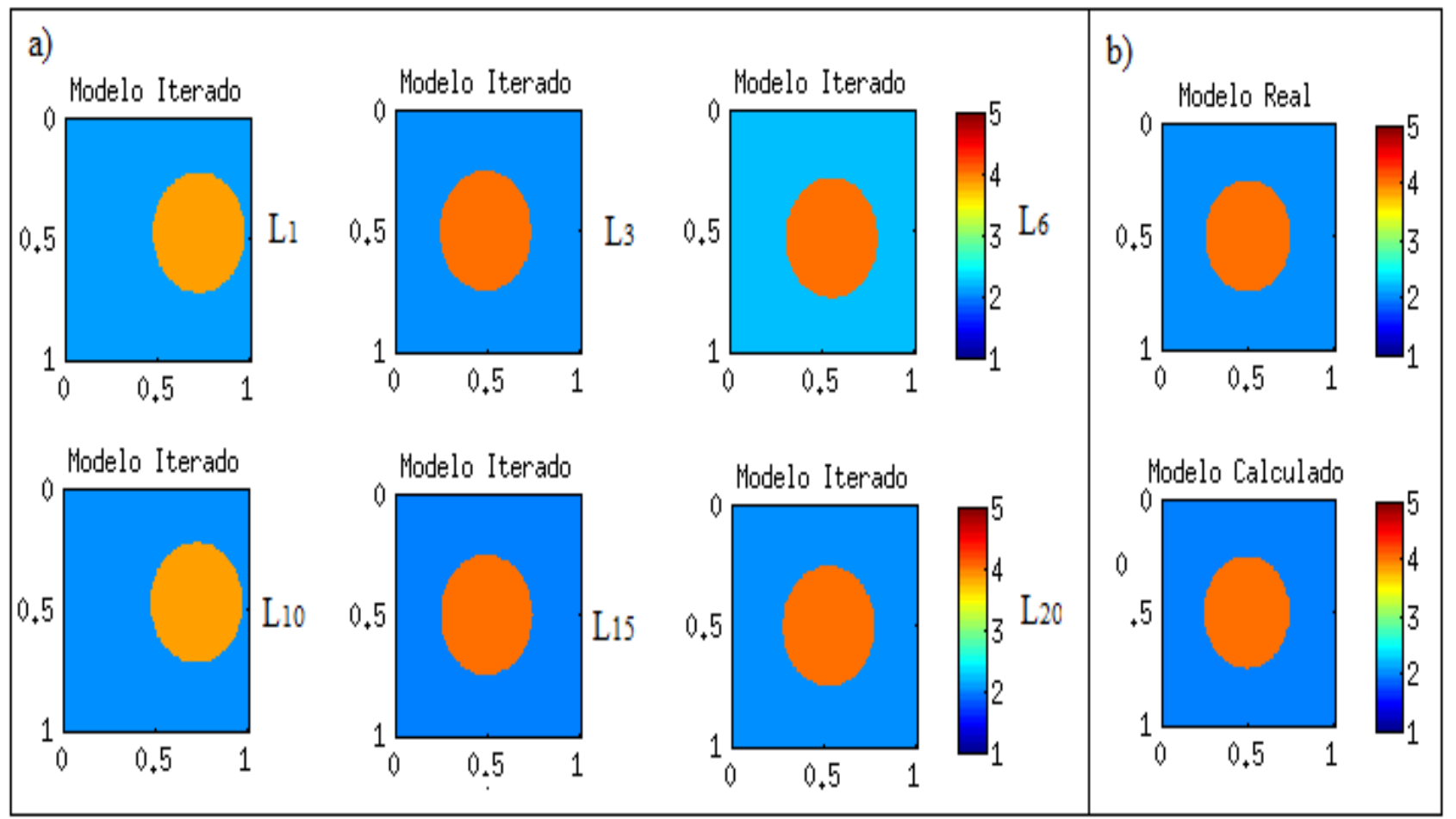

Figura 13: (a) Evolução dos pontos ótimos locais para as seis visitas ao reservatório $A$ $\left(L_{1}, L_{3}, L_{6}, L_{10}, L_{15}\right.$ e $\left.L_{20}\right)$ pelo ANMS; (b) Comparação do modelo real e calculado para o modelo [VHS].

A Figura 14 (a) e (b) mostram instantâneos da propagação do campo de ondas nas camadas do modelo geológico referentes à simulação sísmica do modelo [VHS], $V H S=[1,99974,00780,49320,5002]$, para as janelas de tempo $t=0,28 s$ e $t=0,6 s$, respectivamente. Neste modelo, pode-se identificar o refletor 1 (superior) ocorrido na interface entre as camadas 1 e 2 . E, entre as camadas 2 e 1 , identificou-se o refletor 2 (inferior). 
Observa-se, na Figura 14 (a), a onda refletida e refratada no refletor 1 e na Figura 14 (b), ondas refletidas e refratadas (espalhadas) dentro (e fora) do domo de sal a todo o momento. As Figuras 14 (c) e 14 (d) são representações 3D desses casos, respectivamente.
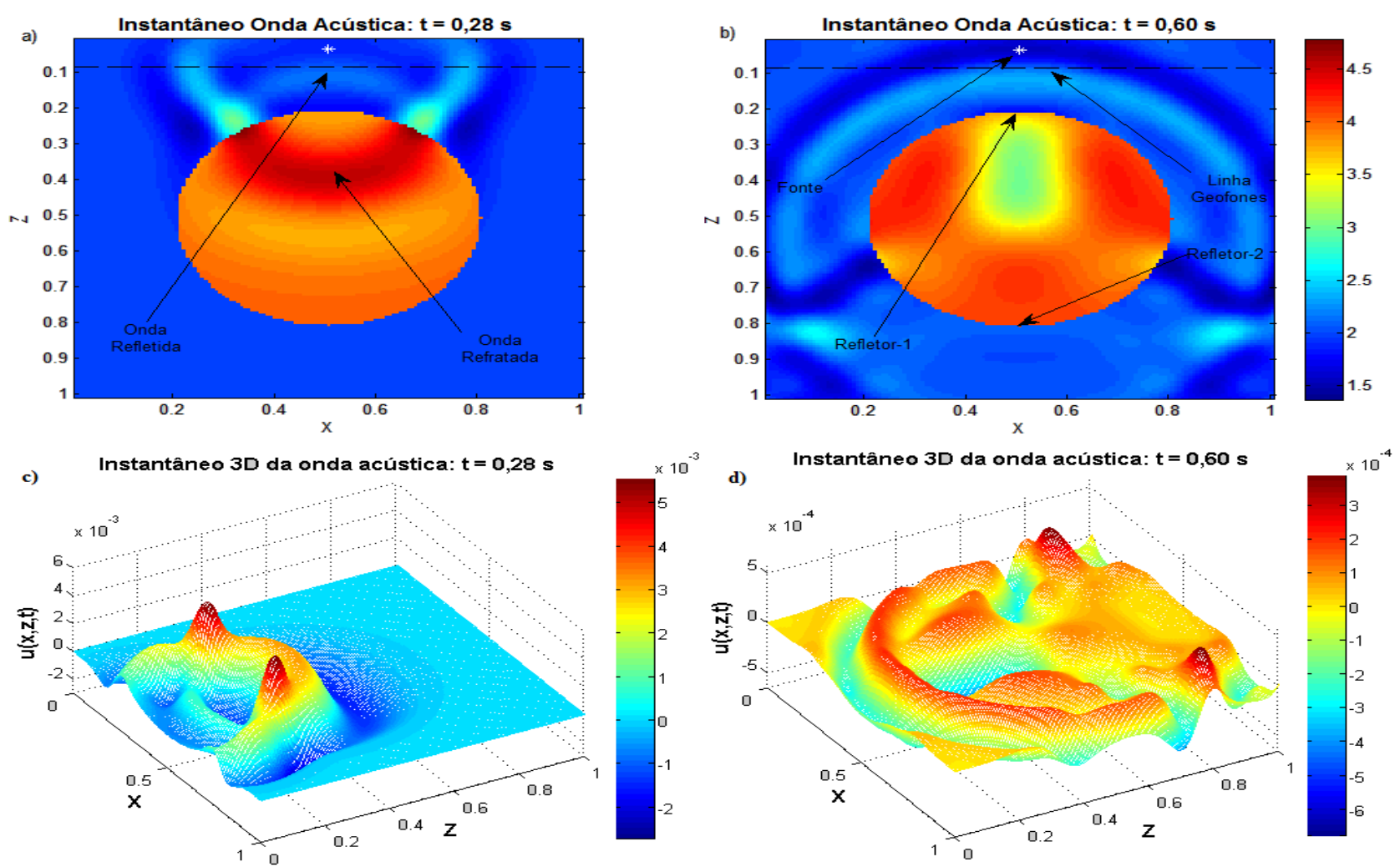

Figura 14: a) e b) Instantâneos da propagação do campo de ondas nas duas camadas do modelo [VHS] para a janela de tempo $t=0,28 \mathrm{~s}$ e $t=0,60 \mathrm{~s}$. As camadas estão dividas pela superfície do domo de sal; c) e d) São representações 3D desses casos.

A Figura 14 (b) mostra os valores de $\phi(\mathbf{m})$ para os vinte simplex(s) otimizados pelo ANMS correspondentes às visitas ao reservatório de modelos $\mathbf{A}$, corrente. Essas Figuras mostram que foram realizadas as 20 visitas permitidas, e que a de número 15 foi a que apresentou menor valor para a função desajuste $\phi(\mathbf{m})$, portanto, considerado o ponto ótimo global da simulação FWI-DFO alcançado pelo ANMS.

A Figura 15 (a) mostra o sismograma para o melhor valor da função custo $\phi(\mathbf{m})$ para o modelo $V H S=[1,99974,00780,49320,5002]$, correspondente à solução ótima, $\phi=0,0513$. A Figura 15 (b) mostra o traço registrado no geofone central ( 71 pontos de malha). Nelas, é possível identificar a assinatura da onda direta e as das reflexões como antes. Observa-se o registro de pequenas reflexões residuais, a partir de $0,7 \mathrm{~s}$, causadas por fenômenos ondulatórios de espalhamento ocorridos no interior do domo de sal (somadas às reflexões residuais das bordas) que são completamente atenuadas com o decorrer do tempo. 

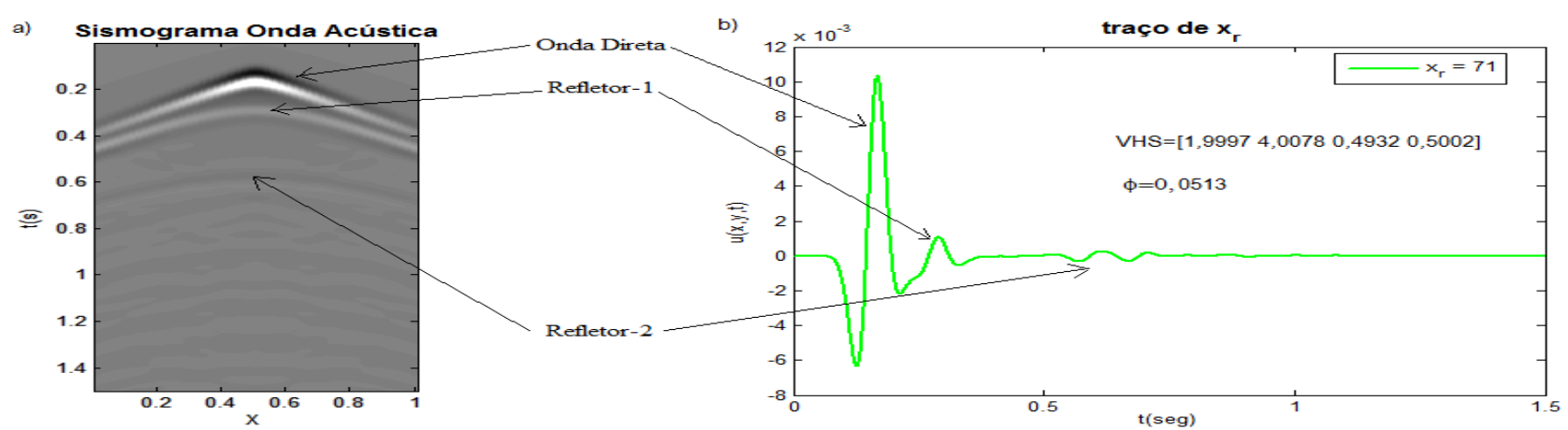

Figura 15: (a) Sismograma para solução ótima; (b) Traço na posição central do sismograma para estes valores no modelo [VHS].

Percebe-se que inversão sísmica recuperou bem os modelos reais para a metodologia FWIDFO empregada aos modelos [V] e [VHS]. Analogamente, os resultados para os outros modelos ([VH], [VHI] e [VHD]) mostraram-se satisfatórios e seguem o mesmo comportamento dos dois modelos analisados ([V] e [VHS]).

Os valores para os parâmetros $\left(V_{i}, h_{i}\right)$, com $i=1, . ., n$ e $n$ sendo o número de parâmetros de cada modelo geológico [V], [VH], [VHI], [VHD] e [VHS], estão relacionados de acordo com os dados da Tabela 4.

Tabela 4: Resultados das simulações para todos os modelos analisados.

\begin{tabular}{|c|c|c|c|c|c|c|c|}
\hline $\begin{array}{c}\text { Parâmetros/ } \\
\text { Valores }\end{array}$ & $\mathbf{V}_{1}$ & $\mathbf{V}_{2}$ & $\mathbf{V}_{3}$ & $10 \mathrm{~h}_{1}$ & $10 \mathrm{~h}_{2}$ & $\begin{array}{l}\text { Função } \\
\text { Custo }\end{array}$ & $\begin{array}{l}\text { Tempo } \\
\text { (S) }\end{array}$ \\
\hline \multicolumn{8}{|c|}{ Modelo [V] } \\
\hline Real & 1 & 2 & 4 & --- & --- & --- & --- \\
\hline Ótimo & 1,0000 & 2,0000 & 3,9985 & --- & --- & 0,0047 & 153,74 \\
\hline \multicolumn{8}{|c|}{ Modelo [VH] } \\
\hline Real & 1 & 2 & 4 & 3,3333 & 6,6667 & --- & --- \\
\hline Ótimo & 1,0000 & 1,9997 & 3,9903 & 3,3191 & 6,6600 & 0,0160 & 579,69 \\
\hline \multicolumn{8}{|c|}{ Modelo [VHI] } \\
\hline Real & 2 & 4 & $\begin{array}{ll}--- \\
\end{array}$ & 3,0000 & 7,0000 & --- & --- \\
\hline Ótimo & 2,0001 & 3,9567 & --- & 2,9988 & 7,0151 & 0,0420 & 338,98 \\
\hline \multicolumn{8}{|c|}{ Modelo [VHD] } \\
\hline Real & 1 & 4 & --- & 5,0000 & 5,0000 & $\begin{array}{l}-- \\
--\end{array}$ & --- \\
\hline Ótimo & 1,9998 & 4,0007 & --- & 5,0399 & 5,0265 & 0,0047 & 510,70 \\
\hline \multicolumn{8}{|c|}{ Modelo [VHS] } \\
\hline Real & 2 & 4 & --- & 5,0000 & 5,0000 & --- & --- \\
\hline Ótimo & 1,9997 & 4,0078 & --- & 4,9321 & 5,0021 & 0,0513 & 619,07 \\
\hline
\end{tabular}


Essa Tabela 4 mostra os resultados dos valores dos parâmetros $V_{1}, V_{2}, V_{3}, h_{1}$ e $h_{2}$ reais e calculados - ótimo global, valor da função objetivo $\phi(\mathbf{m})$ e seu respectivo tempo computacional. Para todos os modelos analisados, os resultados mostram que as simulações em inversão sísmica com base no algoritmo FWI-DFO, desenvolvido nesta pesquisa, conseguiram determinar os parâmetros de cada modelo em um tempo computacional consistente para tolerância adotada. Os cinco modelos geológicos foram construídos com complexidades crescentes, apresentando efeitos de refração, reflexão, difração ([VHD]) e espalhamento ([VHS]). Mesmo assim, os resultados mostram que a metodologia híbrida de otimização, FWI-DFO, aqui desenvolvida, mostrou-se bastante eficaz para todos eles.

Alguns trabalhos foram realizados nesta direção e motivou a pesquisa aqui desenvolvida. Ao realizar comparação dos resultados obtidos nesta análise, tanto para o modelo [V] quanto para o [VH], com os resultados de outros autores, que utilizam otimização global, como por exemplo, Lima (2006), modelos de duas (2) camadas, e Tran \& Hiltunen (2012), modelos de quatro (4) camadas, observou-se uma maior precisão e menor tempo computacional.

Lima (2006), apesar de trabalhar com modelos semelhantes, diferentemente desta pesquisa, realizou estudo com duas camadas, contendo dois parâmetros cada (velocidade e densidade) e considerou as interfaces entre elas fixas e não como parâmetros de modelo, ou seja, um modelo tipo [V]. Esse processo simplifica bastante o problema, diminuindo a complexidade do algoritmo.

Tran \& Hiltunen (2012) utilizaram um número maior de parâmetros, o que é uma vantagem em relação à precisão, mas uma desvantagem em relação ao tempo computacional. Mesmo utilizando a equação de onda elástica em sua modelagem, tornando-a mais realista, pois incorporam os fenômenos da onda transmitida, ondas-S, a precisão ainda é inferior e o tempo computacional é 46 vezes maior.

Comparando os resultados obtidos para o modelo [VHS], mas que podem ser estendidos ao modelo [VHD], com os apresentados em Gauthier, Virieux e Tarantola (1986), bem como o de Van Leeuwen e Herrmann (2013), é possível entender porque os autores deste trabalho escolheram um método de otimização global e não procuraram aperfeiçoar os métodos tradicionais que utiliza cálculo de derivadas como os demais autores têm feito. Especificamente, para o [VHS], ou Camembert, como é mais conhecido, o nosso resultado é visivelmente superior, com o referido modelo diferindo apenas em escala. Entretanto, utilizaram uma modelagem direta no domínio da frequência, equação de Helmholtz, que é muito mais rápida que a modelagem no domínio do tempo.

Também, no caso do Camembert, os autores utilizaram múltiplas fontes, ou seja, maior precisão na amostragem do meio, tanto para o experimento de reflexão quanto no de tomografia sísmica, apenas este último conseguiu um resultado que pode ser aceitável, mas com uma 
precisão muito menor em relação ao que foi obtido pela técnica aqui apresentada, além do tempo computacional ser da ordem de 11 vezes maior.

Resumindo, a metodologia empregada nesta pesquisa apresenta bons resultados, além de abordar um gama de modelos envolvendo um conjunto misto de parâmetros (velocidades e posições de refletores), utiliza-se de um conjunto de técnicas simples de otimização com base em DFO (RJT- CRS - ANMS). O algoritmo gerado é de fácil implementação e resolve o PIS com relativa facilidade, precisão e robustez frente a outras técnicas presentes na literatura. Outro ponto a ser considerado é que, diferentemente da maioria das metodologias vigentes, este algoritmo encontra o mínimo global do PIS por meio do ANMS, que é uma técnica de otimização local. Portanto, menos dispendioso computacionalmente.

\section{CONCLUSÕES E SUGESTÕES}

Neste trabalho, utilizou-se a equação de onda acústica em duas dimensões (2D) no domínio do tempo para modelar o campo de propagação da onda sísmica em um meio geológico. Além disso, usou-se técnicas baseadas em DFO como método de otimização não linear para substanciar a inversão sísmica. Para tanto, foi desenvolvido o algoritmo, FWI-DFO, que resolve o problema direto sísmico (PDS) pelo FDM e utiliza-se do método híbrido de otimização DFO = RJTCRS-ANMS como estratégia para minimizar a função objetivo $\phi(\mathbf{m})$, que quantifica o desajuste entre os dados calculados e os observados pela norma $l_{2}$ no contexto do método dos mínimos quadrados para resolver o problema inverso sísmico (PIS).

Tecnicamente, a estratégia se constitui em, a cada instante, o CRS explora e reduz o espaço de busca dos modelos factíveis ao problema em busca de seu ótimo global, enquanto que o ANMS escolhe aleatoriamente simplex(s). Nesse espaço, cada vez mais reduzido, que será transformado em busca de seu ótimo local, o que se espera é que o ótimo local alcançado pelo ANMS coincida com o ótimo global do PIS. Resumindo, a estratégia do algoritmo híbrido é balancear automaticamente as buscas globais e locais procuradas iterativamente pelo CRS e ANMS, respectivamente.

Para a aplicação da metodologia FWI-DFO, foram construídos cinco modelos de terra ([V], [VH], [VHI], [VHD] e [VHS]), sintéticos, com grau de complexidade crescente envolvendo os principais fenômenos presente na propagação de ondas em meios não homogêneos como efeitos de refração, reflexão, difração e espalhamento. Os resultados encontrados utilizando a técnica DFO (RJT-CRS-ANMS) mostraram uma ótima concordância com os modelos geológicos reais (sintéticos), empregados, e os tempos computacionais encontraram-se dentro dos valores esperados para tolerância aqui adotada.

Especificamente, foi possível identificar, também, que os valores para os parâmetros do modelo são menos sensíveis do que os da função objetivo e que o algoritmo encontra maiores 
dificuldades de convergência com relação aos parâmetros correspondentes para as camadas mais distantes da fonte sísmica. Quanto aos efeitos de difração e espalhamento desenvolvidos nos modelos [VHD] e [VHS], respectivamente, não apresentaram maiores dificuldades nos resultados finais da inversão.

Apesar da não trivialidade do PIS, a metodologia FWI-DFO, aqui empregada, mostrou-se bastante eficiente e adequada às simulações em meios geológicos representados por modelos geométricos simples, cuja construção tomou como base modelos de velocidades de camadas. A aplicação desta técnica em modelos cada vez mais complexos, com mais camadas e torços, sem acarretar um custo computacional excessivamente maior, exige a aplicação de técnicas de redução de parâmetros de modelos. Nesse caso, faz-se necessária a utilização de múltiplas fontes e da modelagem direta no domínio da frequência, com o uso da equação de Helmholtz. Esse posicionamento pode direcionar o desenvolvimento dos próximos trabalhos nesta área de pesquisa.

\section{AGRADECIMENTOS}

Os autores agradecem ao CISCPET, CNPq, FINEP, CTPETRO, FAPERN, CAPES, UFRN, IFRN, IFCE e à Rede Cooperativa de Pesquisa em Geofísica de Exploração pelo apoio financeiro a este trabalho.

\section{REFERÊNCIAS}

Alford, R., Kelly, K., Boore, D. (1974). Accuracy of finite-difference modeling of the acoustic wave equation. Society of Explorations Geophysicists, 39 (6), 834-842. doi:10.1190/1.1440470.

Almeida, V. T. X. D. (2013). Tomografia sísmica de superfície: modelagem direta calculando as trajetórias de ondas refratadas. (Dissertação de mestrado, Universidade Federal Fluminense). Recuperado de http://www.isis.uff.br/wpcontent/uploads/2014/01/dissertacao_victor_almeida.pdf

Bakr, M. (2013). Nonlinear optimization in electrical engineering with applications in matlab. London: The Institution of Engineering and Technology.

Conn, A.R., Scheinberg, K., Vicente, L.N. (2009). Introduction to derivative-free optimization. Society for Industrial and Applied Mathematics. doi: 10.1137/1.9780898718768.

Cunha, M. C. C. (2009). Métodos numéricos. Campinas: UNICAMP.

Diniz-Ehrhardt, M.A. (2010). Métodos sem derivadas para minimização irrestrita. Notas em $\begin{array}{llll}\text { Matemática } \quad \text { Aplicada, } 49 \text { (3), } 87 . & \text { Recuperado de }\end{array}$ https://www.sbmac.org.br/eventos/cnmac/xxxiii_cnmac/pdf/2049.pdf

Diniz-Ehrhardt, M.A., Martinez, J.M., Pedroso, L.G. (2011). Derivative-free methods for nonlinear programming with general lower-level constraints. Computational \& Applied Mathematics, 30 (1), 19-52. Recuperado de http://www.scielo.br/scielo.php?pid=S1807- 
03022011000100003\&script=sci_arttext\&tlng=es

Dourado, W. B., Imada, R. N., Pagamisse, A. (2013). Comparação entre a máscara de nitidez cúbica e o laplaciano para realce de imagens digitais. Anais do Congresso de Matemática Aplicada e Computacional Sudeste. Bauru/SP, Setembro. Recuperado de http://www.sbmac.org.br/cmacs/cmac-se/2013/trabalhos/

Fichtner, A. (2011). Full seismic waveform modelling and inversion. [Springer-Verlag].

doi: 10.1007/978-3-642-15807-0.

Gao, F. Han, L. (2012). Implementing the Nelder-Mead simplex algorithm with adaptive parameters. Computational Optimization and Applications. 51 (1), 259-277. doi: 10.1007/s10589-010-9329-3.

Lagarias, J. C., Reeds, J. A., Wright, M. H., Wright, P. E. (1998). Convergence properties of the Nelder-Mead simplex method in low dimensions. SIAM Journal on Optimization, 9(1), 112147. doi: $10.1137 / \mathrm{s} 1052623496303470$.

Lima, M.V.A.G. (2006). Reflexão sísmica rasa: estimativa de parâmetros elásticos pela inversão dos coeficientes de reflexão da onda $\mathrm{P}$ acima do ângulo crítico. (Dissertação de mestrado Universidade de São Paulo). Recuperado de ftp://www.grec.iag.usp.br/private/adm/katia/teses/agg/m_marcus_vag_lima.pdf

Manzanares-Filho, N., Sousa, B.S., Camacho, R.G.R., Albuquerque, R.B.F., Jorge, A.B. (2007). Airfoil shape optimization using a controlled random search algorithm. Anais do Congresso de THE INTERNATIONAL CONGRESS OF MECHANICAL ENGINEERING, Brasília, DF. Recuperado de http://www.abcm.org.br/app/webroot/anais/cobem/2007/pdf/COBEM2007-2028.pdf

Martins, M.A. (2015). Estratégias para inversão do campo de ondas completo associado a fontes sísmicas reais e virtuais. (Tese de doutorado - Universidade Federal do Rio de Janeiro). Recuperado http://www.coc.ufrj.br/index.php?option=com_docman\&view=document\&alias=2696martins-ma-tm-14-pdf\&category_slug=2015-1\&Itemid=428

Menke, W. (2012). Geophysical data analysis: discrete inverse theory. New York: Academic Press.

Mrinal, K. S. (2006). Seismic inversion. Society of Petroleum Engineers. Richardson, TX: Society of Petroleum Engineers.

Nelder, J. A., Mead, R. (1965). A Simplex Method for Function Minimization. The Computer Journal, 7 (4), 308-313. doi: 10.1093/comjnl/7.4.308.

Odile Gauthier, Jean Virieux, and Albert Tarantola (1986). Two-dimensional nonlinear inversion of seismic waveforms: Numerical results. GEOPHYSICS, 51(7), 1387-1403. doi: 10.1190/1.1442188

Pedroso, L. G. (2005) Sobre o desempenho de métodos de busca direta para minimização irrestrita. (Dissertação de mestrado - UNICAMP). Recuperado de http://repositorio.unicamp.br/handle/REPOSIP/306047

Price, W. L. (1977). A Controlled Random Search Procedure for Global Optimisation. The Computer 
Journal, $20 \quad$ (4), 367-370. Recuperado de https://academic.oup.com/comjnl/article/20/4/367/393971/A-controlled-random-searchprocedure-for-global

Reynolds, A. C. (1978). Boundary conditions for the numerical solution of wave propagation problems. Society of Explorations Geophysicists, 43 (6), 1099-1110. doi:10.1190/1.1440881.

Ricker, N. H. (1940). The form and nature of seismic waves and the structure of seismograms. Geophysics, 5 (4), 348-366. doi: 10.1190/1.1441816.

Rocha Jr., D. C. (2013). Inversão linearizada com regularização para obtenção de velocidades intervalares. (Monografia de Graduação - Universidade Federal da Bahia). Recuperado de http://www.cpgg.ufba.br/gr-geof/geo213/trabalhos-graducao/Daniel-Rocha.pdf

Tarantola, A. (2006). Inverse Problem Theory and Methods for Model Parameter Estimation. Paris: Society for Industrial and Applied Mathematics. doi: 10.1137/1.9780898717921.

Tran, K. T., Hiltunen, D. R. (2012). One-Dimensional Inversion of Full Waveforms using a Genetic Algorithm. Journal of Environmental \& Engineering Geophysics, 17 (4), 197-213. doi: 10.2113/JEEG17.4.197.

Van Leeuwen, T. \& Herrmann, F. J. (2013), Fast waveform inversion without source-encoding. Geophysical Prospecting, 61 (1), 10-19. doi:10.1111/j.1365-2478.2012.01096.x 